\title{
Particle motion driven by solute gradients with application to autonomous motion: continuum and colloidal perspectives
}

\author{
JOHN F. BRADY† \\ Divisions of Chemistry \& Chemical Engineering, and Engineering \& Applied Science, \\ California Institute of Technology, Pasadena, CA 91125, USA
}

(Received 17 March 2010; revised 9 August 2010; accepted 13 August 2010; first published online 3 December 2010)

Diffusiophoresis, the motion of a particle in response to an externally imposed concentration gradient of a solute species, is analysed from both the traditional coarse-grained macroscopic (i.e. continuum) perspective and from a fine-grained micromechanical level in which the particle and the solute are treated on the same footing as Brownian particles dispersed in a solvent. It is shown that although the two approaches agree when the solute is much smaller in size than the phoretic particle and is present at very dilute concentrations, the micromechanical colloidal perspective relaxes these restrictions and applies to any size ratio and any concentration of solute. The different descriptions also provide different mechanical analyses of phoretic motion. At the continuum level the macroscopic hydrodynamic stress and interactive force with the solute sum to give zero total force, a condition for phoretic motion. At the colloidal level, the particle's motion is shown to have two contributions: (i) a 'backflow' contribution composed of the motion of the particle due to the solute chemical potential gradient force acting on it and a compensating fluid motion driven by the long-range hydrodynamic velocity disturbance caused by the chemical potential gradient force acting on all the solute particles and (ii) an indirect contribution arising from the mutual interparticle and Brownian forces on the solute and phoretic particle, that contribution being non-zero because the distribution of solute about the phoretic particle is driven out of equilibrium by the chemical potential gradient of the solute. At the colloidal level the forces acting on the phoretic particle - both the statistical or 'thermodynamic' chemical potential gradient and Brownian forces and the interparticle force - are balanced by the Stokes drag of the solvent to give the net phoretic velocity.

For a particle undergoing self-phoresis or autonomous motion, as can result from chemical reactions occurring asymmetrically on a particle surface, e.g. catalytic nanomotors, there is no imposed chemical potential gradient and the backflow contribution is absent. Only the indirect Brownian and interparticle forces contribution is responsible for the motion. The velocity of the particle resulting from this contribution can be written in terms of a mobility times the integral of the local 'solute pressure' - the solute concentration times the thermal energy - over the surface of contact between the particle and the solute. This was the approach taken by Córdova-Figueroa \& Brady (Phys. Rev. Lett., vol. 100, 2008, 158303) in their analysis of self-propulsion. It is shown that full hydrodynamic interactions can be incorporated into their analysis by a simple scale factor. 
Key words: colloids, Stokesian dynamics, suspensions

\section{Introduction}

The motion of small particles resulting from concentration gradients of chemical solutes is a fundamental process that occurs both in nature and in engineered settings (Anderson 1989). Familiar examples are: diffusiophoresis, osmophoresis, chemotaxis, etc.; even thermophoresis and electrophoresis can be classed in the same general area. Recent interest has focused on 'self-phoresis' or autonomous motion, either in a biological setting or as a result of chemical reactions occurring asymmetrically on a particle surface as with so-called catalytic nanomotors (Paxton et al. 2004, 2006; Hong et al. 2007; Howse et al. 2007; Abecassis et al. 2008; Ibele, Mallouk \& Sen 2009; Kagan et al. 2009; Wang et al. 2009; Ebbens \& Howse 2010). Most treatments of phoretic phenomena model the solute-solvent solution as a Newtonian fluid into which a 'macroscopic' particle is dispersed and then make use of well-established non-equilibrium thermodynamic and continuum hydrodynamic treatments to work out the effect of solute (or other) gradients on the phoretic motion of the particle.

Such a coarse-grained 'continuum-level' approach is quite appropriate in many situations, but it is not the only way to view the problem. A fine-grained 'micromechanical' model in which the solute and the particle are treated on the same footing - as Brownian particles dispersed in a solvent - is also a viable approach and provides a more detailed description of the process. This more detailed approach can offer several advantages. For example, what is the behaviour if the solute is not much smaller in size than the particle? After all, Einstein successfully applied colloidal concepts to a sugar molecule, and in biological applications protein molecules may not be all that much larger than the solute, e.g. another protein. The well-known phenomenon of depletion flocculation (Asakura \& Oosawa 1954) explicitly exploits the finite size of the 'solute' - the depletant. In the continuum approach, the solute has no size at all and so cannot be used to analyse these situations. What about limitations that may arise if the concentration of solute becomes large enough such that solute-solute interactions become important? Concentration effects in multicomponent diffusion - the flux of one species down the concentration gradient of another - is a variant of this situation. What is the physical origin of the potential of interaction between the solute and the particle? In many cases the potential is based on interactions between colloidal particles, not on those with actual solute molecules. Finally, it would be useful to have an independent confirmation that the continuum approach is correct in the appropriate limit. Exactly what is that limit needs to be clarified.

The basic issue we wish to address in this work is the connection between a colloidal description of particle motion due to solute gradients and the more common macroscopic, continuum, treatment. In essence we are asking how the continuum description, in which the solute's concentration is described by a continuous field, emerges from the more primitive (and fundamental) colloidal approach, where the solute is treated as discrete Brownian particles in a viscous fluid.

The motivation for this work is not only to understand this colloidal-continuum connection, but also to address concerns that have been raised about a recent paper on autonomous motion. Córdova-Figueroa \& Brady (2008) modelled the reactioninduced motion of catalytic nanomotors at the colloidal scale and showed that this 
motion could be understood in terms of an imbalance of the local 'solute pressure' exerted by the solute on the particle, an imbalance caused by the distribution of solute resulting from an asymmetric chemical reaction on the particle's surface (an imbalance of the same type that gives rise to depletion flocculation). This approach has been criticized by Jülicher \& Prost $(2009 a, b)$ who, based on a continuum approach, claim that a concentration gradient of solute cannot generate any force on a particle and therefore the analysis of Córdova-Figueroa \& Brady is fundamentally flawed. We show below that the analysis of Córdova-Figueroa \& Brady is in fact correct and, in the appropriate limit, it produces results that are identical to those from the conventional continuum-level modelling of phoretic processes. The misunderstanding expressed by the continuum-level practitioners (Jülicher \& Prost 2009a,b) is resolved by recognizing that there can be two different levels of description of the same process: where a force or interaction appears at one level it may or may not appear in the same context at a different level. For example, in a fluid at the molecular level there are only forces between molecules. At the continuum level, however, there is no net force on a 'fluid point' (unless, of course, there are external forces); rather, the divergence of the continuum stress tensor replaces the effect of the intermolecular forces. What at the fundamental level is a force, is not at the continuum level. This does not mean one approach is right and the other is wrong. They are different levels of description and both should agree in their common (the continuum) domain of overlap. This appears to be the crux of the issue, which we resolve below by explicit, detailed analysis of both the continuum and colloidal perspectives. There also appears to be confusion between a local variation in solute pressure, which is what drives autonomous motion, and a global variation due to a macroscopic concentration gradient of solute that appears in continuum-level treatments of phoretic motion. The micromechanical colloidal analysis separates and clarifies local versus global variations in solute pressure.

In this paper, we refer to the solute's contribution to the total stress in the solution (the mixture of the solvent and the suspended solute) as the 'solute pressure'. Córdova-Figueroa \& Brady (2008) called this solute pressure the 'osmotic pressure' of the solute, as is common in the colloids literature. However, this terminology has led to considerable confusion because the phrase 'osmotic pressure' is often used in different contexts in the physics/chemistry literature and we wish here to avoid confusion as much as possible. In this work, the solute pressure is defined (for dilute solutions) as $\Pi=n k T$, where $n$ is the (local) number density of solute particles and $k T$ is the thermal energy. This definition will be used consistently throughout and the role it plays in diffusiophoresis will be described below. (For non-dilute solute concentrations the solute pressure increases faster than linearly in concentration and involves standard excluded volume and specific interparticle interactions; see Russel, Saville \& Schowalter 1989.)

This paper is organized as follows. In $\S 2$, we provide an overview of the basic problem and a physical discussion of the main results. This section is sufficiently self-contained that readers not interested in the details may read this section and then skip to $\S 6$. The detailed derivation and calculations for the coarse-grained continuum perspective appear next in $\S 3$, while $\S 4$ is devoted to the fine-grained micromechanical colloidal analysis. The treatment of autonomous motion is discussed in $\S 5$. Finally, $\$ 6$ concludes with suggestions on how the colloidal analysis and results can be applied to other phoretic processes such as electrophoresis and surfactant-driven motion at interfaces. 


\section{Overview of the basic problem and the main results}

In the well-known, coarse-grained macroscopic continuum treatment of phoretic motion of a particle of radius $a$ due to an imposed constant concentration gradient of a solute, $\nabla n$, the condition for phoretic motion is that there is no external force acting on the particle. The total force acting on the particle is the sum of the hydrodynamic (actually, the integral of the continuum stress over the particle surface) and the interactive force with the solute. In the absence of any interactive solute force there is no phoretic motion. The imposed concentration gradient of the solute generates a gradient in the solute pressure: $\Pi=n k T$, where $n$ is the number density of solute and $k T$ is the thermal energy. The (global) solute pressure gradient is balanced by a pressure gradient in the solvent (or fluid) so that the macroscopic stress - the sum of the fluid stress and the pressure of the solute - is constant. When there is an interactive force with the solute, however, the hydrodynamic and interactive forces balance and can give rise to a net phoretic motion. In $\S 3$, particular attention is given to the case where the interactive force (or potential) between the particle and solute is short-ranged, so that the notion of a slip velocity at the particle surface is valid. In the simplest case of a short-range, hard-sphere excluded-volume interaction between the solute and the particle characterized by the length $b(\ll a)$, the phoretic velocity is given by the well-known expression

$$
\boldsymbol{U}=-\frac{1}{2} \frac{b^{2}}{\eta} k T \nabla n,
$$

where $\eta$ is the viscosity of the solvent (Anderson 1989).

In the fine-grained colloidal perspective of $\S 4$ both the phoretic particle and the solute are modelled as colloidal particles dispersed in a continuous solvent. The motion of the particles is now governed by the well-established equations of colloidal dynamics (Russel et al. 1989), and we give general expressions valid for all concentrations of solute and for all ratios of the solute size to the phoretic particle size

$$
\lambda=\frac{a_{s}}{a},
$$

where $a_{s}$ is the size of a solute particle. In the micromechanical picture, the fluctuating Brownian behaviour of the solute requires a statistical description and an average over the microstructure - the distribution of solute particles about the phoretic particle. This distribution is governed by the Smoluchowski equation. At this level of description, the particle flux due to Brownian motion in the Smoluchowski equation is the same as if there were statistical or 'thermodynamic' forces acting on the particles, e.g. chemical potential gradient forces. And when we refer to chemical potential gradient or Brownian forces they are to be understood in this statistical sense (see $\S 4)$.

Batchelor $(1976,1983)$ showed that the motion of Brownian particles resulting from an imposed (weak) solute concentration gradient is equivalent to sedimentation in which the 'gravitational' force acting on the particles is the gradient in the chemical potential and the solvent is force-free. The effective chemical potential gradient force acting on the phoretic particle results from the entropic penalty for excluding the solute particle centres from the region occupied by the phoretic particle: $-k T(1+\lambda)^{3} v \nabla n$, where $v=4 \pi a^{3} / 3$ is the volume of the phoretic particle. The force on each solute particle is simply its 'ideal gas' chemical potential gradient, $-k T \nabla \ln n$. (These expressions are valid for a dilute solute and it is important to note, as discussed in $\S 4.1$, that they correspond to a reference frame for the 
(a)

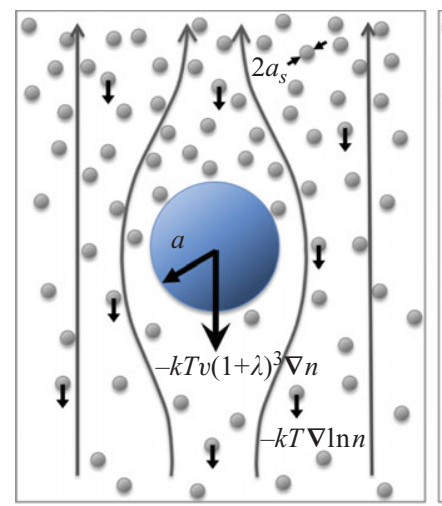

(b)

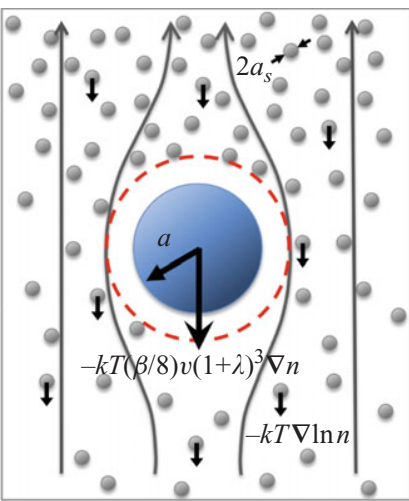

Figure 1. (Colour online) The solute chemical potential gradient force generates a uniform 'back-flow' of fluid so that the volume flux of material at any cross-section is zero. This back-flow precisely compensates the motion of the particle in response to the entropic solute chemical potential gradient force acting on it when there are no specific interactions between the solute and the particle $(a)$. When there are specific interactions, such as the solute being excluded from a region of size $b$ adjacent to the particle, then the back-flow no longer precisely cancels the solute force on the particle and a net phoretic velocity results $(b)$.

motion in which the solvent is force-free.) In response to this entropic force, the

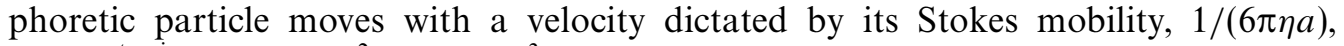
i.e. $\boldsymbol{U}^{\text {entropic }}=-(2 / 9)\left(a^{2} / \eta\right)(1+\lambda)^{3} k T \nabla n$. The force on each solute particle creates a velocity field that decays as $1 / r$ owing to the long-range nature of low-Reynoldsnumber hydrodynamics. By properly summing these long-range interactions (see $\S 4.2$ ), the solute produces a 'back-flow' of fluid that entrains the phoretic particle with a velocity $\boldsymbol{U}^{b f}=+(2 / 9)\left(a^{2} / \eta\right)\left(1+3 \lambda+\lambda^{2}\right) k T \nabla n$, as illustrated in figure 1 . Thus, the net velocity of the phoretic particle is $\boldsymbol{U}=-(2 / 9)\left(a^{2} / \eta\right)\left(2 \lambda^{2}+\lambda^{3}\right) k T \nabla n$, which vanishes in the limit $\lambda \rightarrow 0$.

The colloidal level of description agrees with the continuum perspective of no phoretic velocity in the dual limits of small solute, $\lambda \rightarrow 0$, and dilute solute, $\phi_{s}=n v_{s} \rightarrow 0$, where $v_{s}$ is the volume of a solute particle. This is as expected, for in the continuum perspective the solute has no size and therefore no volume fraction. Although the two analyses agree, they are not the same. At the colloidal level, the phoretic particle experiences a large entropic force proportional to its volume, but the particle's motion in response to this force via its Stokes mobility is precisely compensated by the back-flow of fluid owing to the chemical potential gradient force on all the solute particles. The 'weight' of the solute particles generates an average pressure gradient in the fluid that drives the solvent back up against the solute force. In the continuum perspective, this fluid pressure gradient balances the solute pressure gradient to give a constant macroscopic continuum stress and thus no force on the phoretic particle. In both perspectives, the reference frame for measuring velocity is one of zero volume flux of material.

If there is an interactive force between the phoretic particle and the solute, then the entropic driving force on the phoretic particle now becomes $-k T(\beta / 8)(1+\lambda)^{3} v \nabla n$, where $\beta$ is the second virial coefficient (see (4.6)). No longer is there precise cancellation between the velocity due to this force and the back-flow, and a net velocity results (see figure 1). In the simplest case (which we just take as an example; general formulae are available in $\S 4$ ) when the interactive force is short-ranged and hard-sphere at a 
distance $b$, the net phoretic velocity is

$$
\boldsymbol{U}^{b f}=-\frac{1}{3} \frac{b^{2}}{\eta} k T \nabla n .
$$

(Note that $b$ is the range of the interparticle potential and is not equal to the size of the solute, which is $a_{s}=\lambda a$.) This result has the same form as the continuum prediction (2.1), but the coefficient is $1 / 3$ rather than $1 / 2$. However, it is not that the two perspectives disagree, but rather there is an additional contribution to the velocity of the phoretic particle that must be considered at the colloidal level.

When colloidal particles exert a mutual interactive force on one another, e.g. $\boldsymbol{F}_{1}=-\boldsymbol{F}_{2}=\boldsymbol{F}_{12}(\boldsymbol{r})$, this causes the particles to move relative to one another and thus contributes to the motion of e.g. particle 1, which we associate with the phoretic particle; subscript 2 will denote the solute. Whether this interactive force is due to an actual colloidal interparticle potential, $\boldsymbol{F}_{12}^{(I)}=-\nabla_{1} \Phi$, or due to the relative Brownian motion of the two particles, $\boldsymbol{F}_{12}^{(B)}=-k T \partial \ln P\left(\boldsymbol{x}_{1}, \boldsymbol{x}_{2}\right) / \partial \boldsymbol{x}_{1}=k T \nabla_{r} \ln g$, where $P\left(\boldsymbol{x}_{1}, \boldsymbol{x}_{2}\right)=n_{1} n_{2} g(\boldsymbol{r})$ is the joint probability density of their centres, does not matter. At the Smoluchowski level of colloidal dynamics, the statistical or 'thermodynamic' Brownian force appears as the gradient of the probability density. The total force is $\boldsymbol{F}_{12}^{(I)}+\boldsymbol{F}_{12}^{(B)}=k T \nabla_{r}(\hat{\Phi}+\ln g)$, where $\hat{\Phi}(\boldsymbol{r})$ is the interparticle potential normalized by $k T$ and $\boldsymbol{r}=\boldsymbol{x}_{2}-\boldsymbol{x}_{1}$. The velocity of the phoretic particle due to the interactive and Brownian forces when averaged over the probability distribution of the solute is given by (see $\S 4.5)$

$$
\left\langle\Delta \boldsymbol{U}_{1}\right\rangle=n k T \int\left(\boldsymbol{M}_{11}-\boldsymbol{M}_{12}\right) \cdot \nabla(\hat{\Phi}+\ln g) g \mathrm{~d} \boldsymbol{r},
$$

where $\boldsymbol{M}_{i j}(\boldsymbol{r})$ is the hydrodynamic mobility giving the velocity of particle $i$ due to a force on particle $j$. In equilibrium, $\hat{\Phi}+\ln g=0$, and there is no net average velocity of the phoretic (or any) particle; net motion is a result of the distribution being out of equilibrium. The distribution is driven out of equilibrium by the chemical potential gradient of the solute. In the case of the short-range, hard-sphere force at $b$ in the limit $\lambda \rightarrow 0, \phi_{s} \rightarrow 0$, we show in $\S 4.5 .2$ that

$$
\left\langle\Delta \boldsymbol{U}_{1}\right\rangle=-\frac{1}{6} \frac{b^{2}}{\eta} k T \nabla n .
$$

The total colloidal phoretic velocity agrees precisely with the continuum result: combining (2.5) and (2.3), we get (2.1).

From the colloidal perspective, there are two contributions to the velocity of the phoretic particle: the first comes directly from the solute's chemical potential gradient force acting on the phoretic particle and the associated compensating back-flow of fluid induced by the constant force on all the solute particles. The second is an indirect contribution resulting from the distribution of solute about the phoretic particle being driven out of equilibrium by the solute concentration gradient. It is important to note that in each contribution the total force on the phoretic particle is zero. The total force includes the Stokes drag of the solvent, and so the other forces are balanced by the Stokes drag to give the phoretic particle's velocity. While it gives the same result, the continuum perspective mixes these two contributions into a single term, and therefore it may prove difficult to generalize to other situations such as finite solute size effects or autonomous motion.

In autonomous, as opposed to phoretic motion, which is discussed in $\S 5$, the particle creates its own non-uniform distribution of solute (e.g. via a surface chemical 


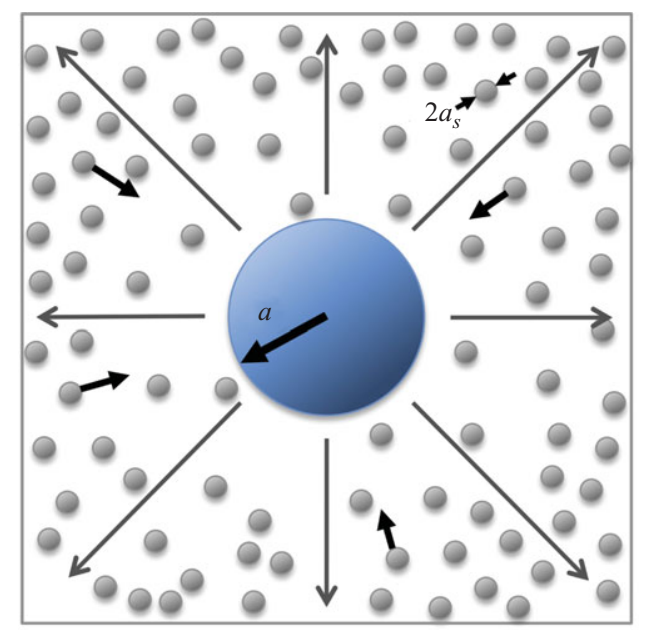

FIGURE 2. (Colour online) The solute concentration distribution due to a reactive particle varies at $1 / r$ and is spherically symmetric (in the far field). The solute particles move radially in response to the chemical potential gradient force acting on them and there is a compensating radial back-flow of fluid so as to conserve volume. This radial flow does not produce any net motion of the reactive particle.

reaction) rather than there being an externally imposed concentration gradient. Since there is no imposed global concentration gradient - no constant chemical potential gradient of solute - the back-flow contribution is absent. To see this, note that far from a reactive particle the disturbance to the uniform concentration of solute, $n^{\infty}$, behaves as $s / r$, where $s$ is the source (or sink) strength of the chemical reaction. Thus, the concentration of solute varies as $-s \boldsymbol{x} / r^{3}$, resulting in a chemical potential gradient force on a solute particle of $+k T\left(s / n^{\infty}\right) x / r^{3}$. This radial force distribution produces no back-flow: the $1 / r$ velocity disturbance due to a force times the $1 / r^{2}$ force density results in a conditionally convergent integral and the radial symmetry produces no net flow as illustrated in figure 2. Higher-order distributions of solute (e.g. dipolar, etc.) decay at least as fast as $1 / r^{2}$, giving a force of $1 / r^{3}$ and a velocity of $1 / r^{4}$, which is absolutely convergent. There is no back-flow; interactions can be added in a pair-wise fashion.

As a result, for autonomous motion only the Brownian and interparticle forces contribution, (2.4), is present; if there is any net motion it can only result from this term. We can gain additional insight by defining the perturbation to the equilibrium distribution, $g=g^{e q}(1+f)=\mathrm{e}^{-\hat{\Phi}}(1+f)$, and integrating (2.4) by parts to obtain

$$
\left\langle\Delta \boldsymbol{U}_{1}\right\rangle=\frac{L\left(r_{c}\right)}{6 \pi \eta a} n^{\infty} k T \oint_{r_{c}} \boldsymbol{n} f \mathrm{~d} S,
$$

where $\boldsymbol{n}$ is the normal pointing out of the particle and the volume integral has been neglected because $\nabla \cdot\left(\boldsymbol{M}_{11}-\boldsymbol{M}_{12}\right) \sim O\left(\lambda^{3}\right)$ and is small as $\lambda \rightarrow 0$ (see (4.59)). In (2.6), we have taken the interparticle potential to be hard-sphere at the contact distance $r_{c}$, and $L\left(r_{c}\right)$ is a non-dimensional hydrodynamic function of the hard-sphere contact distance. The local solute pressure is $\Pi(\boldsymbol{r})=n(\boldsymbol{r}) k T=n^{\infty} k T g(\boldsymbol{r})=n^{\infty} k T(f(\boldsymbol{r})+1)$ and thus (2.6) is

$$
\left\langle\Delta \boldsymbol{U}_{1}\right\rangle=\frac{L\left(r_{c}\right)}{6 \pi \eta a} \oint_{r_{c}} \boldsymbol{n} \Pi(\boldsymbol{r}) \mathrm{d} S .
$$


Equation (2.7) offers a clear physical interpretation: the velocity of the autonomous particle is the 'mobility' of the particle, $L\left(r_{c}\right) /(6 \pi \eta a)$, times the integral of the solute pressure over the surface of contact between the particle and the solute, $\oint_{r_{c}} \boldsymbol{n} \Pi(\boldsymbol{r}) \mathrm{d} S$. If the solute pressure is higher on one side of the particle than the other due to the asymmetric chemical reaction, then there will be a net velocity of the particle. This is the argument (and the analysis) of Córdova-Figueroa \& Brady (2008) in their treatment of the 'osmotic motor' - autonomous motion due to propulsion caused by an asymmetric chemical reaction on the motor surface. From the known behaviour of hydrodynamic interactions between particles, the mobility function is $L\left(r_{c}=a(1+\right.$ $\Delta))=(3 / 2) \Delta^{2}(1+(2 / 3) \Delta) /(1+\Delta)^{3}$, where $\Delta=b / a$. Córdova-Figueroa and Brady took the hard-sphere excluded-volume interparticle force distance $b$ to be of $O(a)$ in which case $L\left(r_{c}\right) \approx 1$ and the reduction in the mobility of the motor due to hydrodynamic interactions between the motor and the solute could be neglected. Including the effects of the solute-motor hydrodynamics gives only a quantitative change to the results of Córdova-Figueroa and Brady - one need only scale their results by the factor $L\left(r_{c}\right)$; all other results and conclusions of their analysis are unchanged. (See $\S 5$ for a summary and extension of their results to include hydrodynamics.)

Not only is the colloidal perspective correct and able to produce all the results of the continuum perspective, but it provides a means to go beyond the coarse-grained continuum mean-field description of such processes. The general formulae presented in $\S 4$ are valid for any size ratio (even if the solute is larger than the phoretic particle), for any interparticle potential and for any volume concentration of solute. Analytical progress may not be possible beyond the dilute limit (i.e. to $O\left(\phi_{s}\right)$ ), but a Stokesian dynamics approach is possible (Brady \& Bossis 1988). The colloidal perspective also provides a rational starting point to go beyond the mean-field description of other phoretic processes such as electrophoresis. Just as the detailed pair- and many-body hydrodynamic interactions can be incorporated at the colloidal level, so too can the pair- and many-body electrostatic interactions necessary to go beyond the Poisson Boltzmann treatment of electrokinetics (Bonnecaze \& Brady 1992).

Having given a general physical description of the behaviour, we now turn to the details. The reader not interested in the details may skip to the conclusions in $\S 6$.

\section{Continuum perspective}

Here we develop the equations employed to model the phoretic motion of small micrometre-sized particles. Similar treatments can be found in books (Anderson 1989; Russel et al. 1989), but a brief review is needed to understand the similarities and differences with the colloidal treatment discussed in $\S 4$.

Consider a solvent into which solute particles (or molecules) are dispersed. We shall refer to this solvent-solute mixture as a 'solution' and reserve the word 'fluid' to mean the solvent into which the solute is dispersed. Both the solvent and the solution are treated as continua. A particle of size $a$ is introduced into the solution. We want to determine the forces acting on the 'large' particle and its subsequent motion. (The particle is 'large' compared to the solute particles.)

The solution is modelled as an incompressible Newtonian-fluid continuum with stress tensor

$$
\boldsymbol{\Sigma}=-p \boldsymbol{I}+2 \eta \mathbf{e},
$$

where the rate of strain tensor $\mathbf{e}=(1 / 2)\left(\nabla v+(\nabla v)^{\dagger}\right)$, with $\boldsymbol{v}$ the solution velocity and $\eta$ its viscosity. The solute will be assumed so dilute that it does not affect the viscosity 
or the form of the constitutive relation. (These restrictions are relaxed in $\S 6$.) The pressure appearing in (3.1) consists of the solvent or fluid pressure $p_{f}$ plus the 'solute' pressure:

$$
p=p_{f}+n k T,
$$

where $n$ is the number density of the solute and $k T$ is the thermal energy. Note that in (3.1) the stress $\boldsymbol{\Sigma}$ and the velocity $\boldsymbol{v}$ are those of the solution - the fluid plus the dilute solute - and are obtained by averaging over a small volume element containing solvent and many solute particles. The dilute solute produces an 'ideal gas' contribution to the solution stress.

The solute particles are carried along with the solution, undergo Brownian motion characterized by a diffusivity $D$ and may be acted upon by a force per particle $f_{s}$, where we have used the subscript ' $s$ ' to denote a solute particle. This force could be an external force such as gravity, but our interest is when this force is an interactive force with the particle as described below. The solute obeys the conservation equation

$$
\frac{\partial n}{\partial t}+\nabla \cdot \boldsymbol{j}=0
$$

where the solute particle flux $\boldsymbol{j}$ is given by the standard expression

$$
\boldsymbol{j}=n \boldsymbol{v}-D\left[\nabla n-n \boldsymbol{f}_{s} / k T\right],
$$

and $D / k T$ is the solute's mobility.

The momentum balance for the solution is Cauchy's equation for a continuum

$$
\rho \frac{\mathrm{D} \boldsymbol{v}}{\mathrm{D} t}=\nabla \cdot \boldsymbol{\Sigma}+n \boldsymbol{f}_{s},
$$

with the body force per unit volume arising from the force on the solute. Conservation of mass for the solution is the usual incompressibility requirement

$$
\nabla \cdot v=0 .
$$

It is important to note that because the solution is incompressible the absolute level of the pressure in the fluid does not matter. The fluid pressure, $p_{f}$, will take on any value or spatial variation so as to ensure that the solution pressure, $p$, guarantees incompressibility.

The total surface or hydrodynamic force exerted on the particle is given by the integral of the continuum stress over the particle surface

$$
\boldsymbol{F}^{\text {hyd }}=\oint \boldsymbol{n} \cdot \boldsymbol{\Sigma} \mathrm{d} S,
$$

where $\boldsymbol{n}$ is the normal pointing out of the particle and into the solution.

Since the forces exerted on the solute particles are due to their interaction with the larger particle (or boundary), there is an equal and opposite force exerted by the solute on the particle

$$
\boldsymbol{F}^{s}=-\int_{V_{\text {out }}} n \boldsymbol{f}_{s} \mathrm{~d} V
$$

where the integral is over the volume of the solution outside the particle. The net force exerted by the solution on the particle is then

$$
\boldsymbol{F}=\boldsymbol{F}^{h y d}+\boldsymbol{F}^{s}=\oint \boldsymbol{n} \cdot \boldsymbol{\Sigma} \mathrm{d} S-\int_{V_{\text {out }}} n \boldsymbol{f}_{s} \mathrm{~d} V .
$$


The condition for phoretic - force-free - motion is that there is no external force exerted on the particle and the particle is small enough that its inertia can be neglected (small Stokes numbers: $S t=\rho_{p} U a / \eta \ll 1$, where $\rho_{p}$ is the density of the particle and $U$ its (to-be-determined) velocity), and thus

$$
\boldsymbol{F}=0 \text {. }
$$

Note that phoretic motion corresponds to zero total force acting on the particle a balance of hydrodynamic shearing forces at the particle surface with the solute interactive forces. (In general, one should include a condition of zero torque, but this is not needed for the spherical particles considered here.) The motion does not correspond to one of zero hydrodynamic force, although it may appear to be so in the 'thin interfacial layer' approximation discussed below. With this continuum perspective, we are now in a position to discuss the phoretic motion of a particle.

\subsection{External solute gradient, no interactive potential}

The first problem we consider is a neutral sphere of radius $a$ immersed in a concentration gradient of solute. By neutral we mean that the particle exerts no force on the solute, $\boldsymbol{f}_{s}=0$. We shall neglect the effects of inertia - low-Reynoldsnumber $(R e=\rho U a / \eta \ll 1)$ flows - so the momentum balance for the solution is simply the Stokes equations

$$
0=-\nabla p+\eta \nabla^{2} v, \quad \nabla \cdot v=0
$$

with boundary conditions

$$
\begin{gathered}
\boldsymbol{v} \sim 0, \quad p \sim 0 \quad \text { as } \quad r \rightarrow \infty, \\
\boldsymbol{v}=\boldsymbol{U} \quad \text { at } \quad r=a,
\end{gathered}
$$

where $\boldsymbol{U}$ is the 'unknown' phoretic velocity of the particle. (The pressure at infinity can be set to an arbitrary constant.) The origin of the coordinate system is at the centre of the moving particle. The solution to the above is the standard Stokes flow outside a translating sphere with $\oint \boldsymbol{n} \cdot \boldsymbol{\Sigma} \mathrm{d} S=\boldsymbol{F}^{\text {hyd }}=-6 \pi \eta a \boldsymbol{U}$. The requirement of no net force on the particle from (3.9) gives, as expected, no phoretic motion $\boldsymbol{U}=0$, and no solution motion $v=0, p=0$.

We have, of course, arrived at the expected result, but it is instructive to examine the solute distribution and to see how this non-uniform distribution is balanced mechanically. In a frame of reference moving with the particle the solute satisfies

$$
\frac{\partial n}{\partial t}+\boldsymbol{v}^{\prime} \cdot \nabla n=D \nabla^{2} n
$$

where $\boldsymbol{v}^{\prime}=\boldsymbol{v}-\boldsymbol{U}$. The boundary conditions are no flux through the particle surface and a uniform solute concentration gradient far from the particle,

$$
\begin{gathered}
\boldsymbol{n} \cdot \nabla n=0 \quad \text { at } \quad r=a, \\
n \sim n^{\infty}+\boldsymbol{x} \cdot \nabla n^{\text {ext }} \quad \text { as } \quad r \rightarrow \infty,
\end{gathered}
$$

where $\boldsymbol{x}$ is the position vector (of magnitude $r=|\boldsymbol{x}|$ ) from the particle centre, $n^{\infty}$ is the constant level of the solute and $\nabla n^{\text {ext }}$ is the constant imposed concentration gradient of solute. At steady state and for small Péclet numbers (see below) the left-hand side of (3.14) is zero and the solution for $n$ is simply

$$
n=n^{\infty}+\boldsymbol{x} \cdot \nabla n^{\text {ext }}\left(1+\frac{1}{2} \frac{a^{3}}{r^{3}}\right) .
$$


This non-uniform solute concentration field generates a gradient in solute pressure $\nabla \Pi=k T \nabla n(\boldsymbol{x})=k T \nabla n^{e x t} \cdot\left(\boldsymbol{I}+(1 / 2)(a / r)^{3}\left(\boldsymbol{I}-3 \boldsymbol{x} \boldsymbol{x} / r^{2}\right)\right)$, which is balanced by the fluid pressure, $p_{f}$, such that the solution pressure, $p$, is a constant, a requirement for the incompressibility of the solution. Note that from (3.11) the pressure satisfies Laplace's equation, $\nabla^{2} p=0$, as does the concentration field, $\nabla^{2} n=0$, implying the solvent or fluid pressure also satisfies Laplace's equation, $\nabla^{2} p_{f}=0$.

\subsection{External solute gradient with interactive potential}

In this section, we repeat the above problem but now allow for an interactive potential between the solute and the particle. The primary interest is in situations where the interactive potential is short-ranged, as this leads to the notion of a 'slip' velocity at the particle surface. We take the length scale of the interactive potential to be $\delta$ and require $\delta / a \ll 1$. The problem is most straightforwardly solved by matched asymptotic expansions with an 'outer' region, where the solute body force is zero, and 'inner' region adjacent to the particle surface of thickness $\delta$, where the solute body force is not zero.

\subsubsection{Outer region: $r \sim O(a)$}

Since the solute body force is zero $\left(f_{s}=0\right)$ in the outer region the solution satisfies the same Stokes equations (3.11), but with a slip velocity at the particle surface

$$
\boldsymbol{v}=\boldsymbol{U}+\boldsymbol{v}^{\text {slip }}, \quad \text { as } r \rightarrow a^{+},
$$

where we need to determine the slip velocity, $\boldsymbol{v}^{\text {slip }}$, and the condition is one of matching to the inner solution.

Before proceeding to the inner region, it is helpful to examine the expression for the force on the particle. In (3.9) the surface integral is over the actual surface, $r=a$, of the particle. By application of the divergence theorem the integral of $\boldsymbol{\Sigma}$ can be transformed to a surface just outside the 'interfacial layer' $\delta$

$$
\oint_{r=a} \boldsymbol{n} \cdot \boldsymbol{\Sigma} \mathrm{d} S=\oint_{r>a+\delta} \boldsymbol{n} \cdot \boldsymbol{\Sigma} \mathrm{d} S+\int_{a<r<a+\delta} n \boldsymbol{f}_{s} \mathrm{~d} V,
$$

where we have made use of Cauchy's equation of motion (3.5) for low Reynolds numbers (no inertia). Thus, the total force (3.9) on the particle becomes

$$
\boldsymbol{F}=\oint_{r>a+\delta} \boldsymbol{n} \cdot \boldsymbol{\Sigma} \mathrm{d} S
$$

The reciprocal theorem for the Stokes flow problem in the outer region allows us to write in the limit $\delta / a \rightarrow 0$

$$
\boldsymbol{F}=-6 \pi \eta a \boldsymbol{U}-\frac{3}{2} \frac{\eta}{a} \oint_{r \rightarrow a^{+}} \boldsymbol{v}^{\text {slip }} \mathrm{d} S .
$$

The condition for phoretic motion of zero total force gives the phoretic velocity

$$
\boldsymbol{U}=-\frac{1}{4 \pi a^{2}} \oint_{r \rightarrow a^{+}} \boldsymbol{v}^{\text {slip }} \mathrm{d} S
$$

The phoretic velocity is the average slip velocity associated with the interactive forces between the solute and the particle that are localized in a thin interfacial layer of thickness $\delta \ll a$. To complete the determination of the phoretic velocity, one needs to determine the slip velocity and the solute concentration at the actual particle surface $r=a$, which requires solving the problem in the inner region. The solute concentration 
field in the outer region is the same as before (3.17) because the matching condition on the solute flux is $\boldsymbol{n} \cdot \boldsymbol{j}^{\text {out }}=\boldsymbol{n} \cdot \boldsymbol{j}^{\text {in }}=0$. (We are assuming that the build-up of concentration in the interfacial region is not so large that the normal flux from the outer region is not zero, but rather is balanced by a tangential flux along the surface. This more general boundary condition can also be addressed.)

\subsubsection{Inner region: $Y=(r-a) / \delta \sim O(1)$}

In the inner region near the particle surface the radial coordinate is scaled with the interfacial layer thickness, $Y=(r-a) / \delta$, and radial derivatives are larger than derivatives along the surface. The solute conservation equation at steady state is simply

$$
\frac{\partial j_{Y}^{i n}}{\partial Y}=0
$$

or, since the normal flux at the actual particle surface is zero,

$$
j_{Y}^{i n}=-\frac{D}{\delta}\left(\frac{\partial n^{i n}}{\partial Y}+n^{i n} \frac{\partial \hat{V}}{\partial Y}\right)=0,
$$

where we have taken the solute force to be derivable from a potential, $f_{s}=-\nabla V$, and have non-dimensionalized the solute potential with the thermal energy, $k T$. The concentration distribution within the interfacial layer is given by the Boltzmann distribution

$$
n^{\text {in }}=n^{\text {out }}\left(\boldsymbol{x}_{\|}\right) \mathrm{e}^{-\hat{V}},
$$

where $\boldsymbol{x}_{\|}$denotes the coordinates parallel to the surface of the particle and $n^{\text {out }}\left(\boldsymbol{x}_{\|}\right)$ is the solute concentration distribution at the particle surface from the outer solution (3.17).

The momentum balance in the direction normal to the surface to leading order in $1 / \delta$ is

giving

$$
0=-\frac{\partial p^{i n}}{\partial Y}-n^{i n} k T \frac{\partial \hat{V}}{\partial Y}
$$

$$
p^{\text {in }}=n^{\text {out }} k T\left(\mathrm{e}^{-\hat{V}}-1\right) .
$$

Outside the interfacial layer $\hat{V}=0$, and $p^{\text {in }}$ vanishes because it is of larger magnitude in the inner region and thus must match to zero. This also guarantees that the parallel or slip velocity is finite (see below). In the inner region the pressure scales as $p^{i n} \sim O(n k T)$. The normal or $Y$-component of the solution velocity is a factor of $\delta / a$ smaller than the parallel component by continuity. And the parallel component will be seen below to scale as $\left|\boldsymbol{v}_{\|}\right| \sim O\left(n k T \delta^{2} /(\eta a)\right)$. The small value of the slip velocity is why we can neglect the viscous stress, $(\eta / \delta) \partial^{2} v_{Y} / \partial Y^{2}$, compared to the pressure gradient and body forces in the $Y$ momentum balance (3.26). The pressure outside the inner region scales as in a Stokes flow $p^{\text {out }} \sim O(\eta v / a) \sim O\left(n k T(\delta / a)^{2}\right)$ and thus is smaller than the pressure in the inner region, leading to the requirement that $p^{i n} \rightarrow 0$ as $Y \rightarrow \infty$.

The momentum balance parallel to the surface is to leading order

$$
0=-\nabla_{\|} p^{i n}+\eta \frac{1}{\delta^{2}} \frac{\partial^{2} \boldsymbol{v}_{\|}}{\partial Y^{2}},
$$

where $\boldsymbol{v}_{\|}$denotes the velocity components parallel to the particle surface. The boundary conditions on $\boldsymbol{v}_{\|}$are no-slip at the particle surface: $\boldsymbol{v}_{\|}=\boldsymbol{U}_{\|}$at $Y=0$, 
and a finite slip velocity as $Y \rightarrow \infty$. Here, $\boldsymbol{U}_{\|}=\boldsymbol{U} \cdot(\boldsymbol{I}-\boldsymbol{n n})$ is the parallel component of the particle's sought-after phoretic velocity. Using (3.27) for $p^{\text {in }}$ in (3.28), the parallel velocity is

$$
\boldsymbol{v}_{\|}=\boldsymbol{U}_{\|}-\frac{\delta^{2}}{\eta} k T \nabla_{\|} n^{\text {out }}\left[\int_{0}^{Y} \xi\left(\mathrm{e}^{-\hat{V}}-1\right) \mathrm{d} \xi+Y \int_{Y}^{\infty}\left(\mathrm{e}^{-\hat{V}}-1\right) \mathrm{d} \xi\right],
$$

giving a slip velocity as $Y \rightarrow \infty$ of

$$
\boldsymbol{v}^{\text {slip }}=-\frac{\delta^{2}}{\eta} \zeta k T \nabla_{\|} n^{\text {out }},
$$

where $\zeta$ is the non-dimensional integral of the interactive potential

$$
\zeta=\int_{0}^{\infty} \xi\left(\mathrm{e}^{-\hat{V}(\xi)}-1\right) \mathrm{d} \xi
$$

The above result for the slip velocity and $\zeta$ agree precisely with those of Anderson (1989). Note that since $\boldsymbol{n} \cdot \nabla n^{\text {out }}=0$ from the outer problem, $\nabla_{\|} n^{\text {out }}$ in (3.29) can be replaced by $\nabla n^{\text {out }}$.

From the solution for the concentration field in the outer region (3.17) at the surface $a^{+}$we have

and

$$
n^{\text {out }}=n^{\infty}+\frac{3}{2} \boldsymbol{x} \cdot \nabla n^{\text {ext }},
$$

$$
\nabla_{\|} n^{\text {out }}=\frac{3}{2} \nabla n^{e x t} \cdot[\boldsymbol{I}-\boldsymbol{n} \boldsymbol{n}] .
$$

Using the above for the concentration field, the phoretic velocity of the particle from (3.22) is

$$
\boldsymbol{U}=\frac{\delta^{2}}{\eta} \zeta k T \nabla n^{e x t}
$$

The phoretic velocity can be either up or down the concentration gradient of the solute depending on the sign of $\zeta$. For attractive potentials, $\mathrm{e}^{-\hat{V}(\xi)}-1>0$, and the slip velocity (3.29) is in the direction of lower solute concentration. For attractive potentials the concentration of solute is higher in the interfacial layer, resulting in a pressure gradient from (3.27) parallel to the interface that drives the solution down the concentration gradient; the particle then moves in the opposite direction - up the concentration gradient - so that the net force on the phoretic particle is zero. And just the opposite occurs for repulsive potentials. From a thermodynamic perspective, the phoretic particle moves to lower its free energy by going towards (away from) attractive (repulsive) interactions with the solute.

To make further progress a model for the interaction potential between the solute and the particle is needed. Here, we take the simplest case of a hard-sphere interaction, where the potential is zero beyond a length $b$ and infinite for distances less than $b$. Thus, $\zeta=-(b / \delta)^{2} / 2$ and the phoretic velocity becomes

$$
\boldsymbol{U}=-\frac{1}{2} \frac{b^{2}}{\eta} k T \nabla n^{e x t}
$$

Note that $b$ is not the radius of a solute particle; in the continuum limit the solute has no size and is fully characterized by its diffusivity, $D$, number density, $n$, and interactive force, $\boldsymbol{f}_{s}$.

If the interaction potential between the solute and the particle is not short-ranged, then no simple interfacial layer analysis is possible and the solution velocity and solute 
concentration fields are fully coupled and must be solved together. Note, however, that one can always proceed to obtain (3.20), it is just that the length $\delta$ beyond which one can set the hydrodynamic force equal to zero is no longer near the actual particle surface and thus no simplifications arise.

As a representative magnitude of the phoretic velocity, for a $1 \mu \mathrm{m}$ particle with an excluded-volume interactive length $b \approx 10 \mathrm{~nm}$ in water at room temperature for a concentration gradient of $10^{3} \mathrm{M} \mathrm{m}^{-1}$ (corresponding to a $1 \mathrm{mM}$ (one millimolar) solute solution varying over the particle size), (3.35) gives $U \approx 1 \mu \mathrm{m} \mathrm{s}^{-1}$.

As a last remark, advection of the solute was neglected because the Péclet number was assumed small; $P e=U a / D \sim(k T / \eta) \delta^{2} \zeta a \nabla n^{\text {ext }} / D \ll 1$. For the motion in the interfacial layer the appropriate Péclet number is smaller still by a factor of $(\delta / a)^{2}$.

\section{Colloidal perspective}

The continuum analysis is fine as a coarse-grained approach, but there are many reasons to seek a more detailed description of the phoretic process. First, it would be useful to have an independent confirmation that the continuum approach is correct in the appropriate limit. Exactly what is that limit needs to be clarified. But there are other questions one would like to address. For example, what is the behaviour if the solute is not much smaller in size than the particle? In the continuum approach the solute has no size at all. Said differently, how does the behaviour of a very large particle emerge from its colloidal modelling - passing to a second 'continuum' limit when the large particle is much larger than the others and so appears as a macroscopic object? What about limitations that may arise if the concentration of the solute becomes so large that solute-solute interactions become important? How do hydrodynamic interactions between the solute and the particle influence the behaviour? And finally, what is the physical origin of the potential of interaction between the solute and the particle? In many cases the potential is based on interactions between colloidal particles - that is, the solute is modelled as a (smaller) colloidal particle itself. To answer these questions a more detailed model is required. And we shall see that understanding how hydrodynamic interactions enter the picture is key.

To provide a more detailed approach, we model both the phoretic particle and the solute as colloidal particles immersed in a molecular solvent that will be treated as a continuum. As before, we shall refer to the solvent as the 'fluid' and the solvent containing the dispersed colloidal particles as a 'suspension'. And we shall make precise in $\S 4.3$ the connection between the 'solution' of the continuum perspective and the 'suspension' here.

Two important issues need to be addressed at the colloidal level. (i) The motion of particles relative to the surrounding fluid results in hydrodynamic interactions among the particles, and at low-Reynolds-numbers hydrodynamic interactions are long-ranged and require care when determining interactions in an unbounded suspension. Further, colloidal particles undergo Brownian motion, which also involves hydrodynamics, and which requires a statistical description for the probability of finding particles in a particular configuration; this probability density is governed by the Smoluchowski equation. (ii) The 'forces' causing the particles to move are of two types. First, global or macroscopic concentration gradients are accompanied by chemical potential gradients that exert statistical or 'thermodynamic' forces on the colloidal particles. There are also thermodynamic forces exerted on the solvent molecules in response to the solute's chemical potential gradient - the two are linked by the Gibbs-Duhem equation. What is needed at the colloidal level, however, is the 
motion of the colloidal particles relative to the solvent, and as discussed in $\S 4.1$ this relative motion is the same as if there are certain steady forces acting on the colloidal particles and the solvent is force-free. And secondly, there may be interactive forces, e.g. London-van der Waals, steric, etc., between the colloidal particles themselves that, along with the relative Brownian motion of the particles, can result in net motion of the phoretic particle.

The starting point for the analysis is two papers by Batchelor $(1982,1983)$ on sedimentation and diffusion in polydisperse suspensions. Indeed, all the necessary information is contained in these two papers, although extracting the needed formulae and the appropriate limits requires careful reading. The derivation given below is designed to bring out the necessary features and spare the reader the need to dissect these two papers. The chemical potential gradient driving forces are discussed first in $\S 4.1$, followed by a discussion of the proper summation of the long-range hydrodynamic interactions in $\S 4.2$. The connection between the continuum and colloidal perspectives is made precise in $\S 4.3$. The chemical potential gradient driving forces are then combined with the long-range hydrodynamic interactions in $\S 4.4$ to give the 'back-flow' contribution to the phoretic velocity. The motion due to the interactive and Brownian forces is discussed in $\S 4.5$.

\subsection{Chemical potential gradient driving force}

Motion resulting from a concentration gradient of solute is driven by the gradient in the chemical potential of the solute. Consider a suspension of composed of $k$ different species. When the suspension is out of equilibrium each particle of species $k$ experiences a force $-\nabla \mu_{k}$, where $\mu_{k}$ is its chemical potential. This 'force' is statistical in nature - an average particle in an ensemble of systems will experience this force. (The forces on an individual particle in an individual system is a different matter and requires a finer level of description than that used, or needed, here to describe the motion of colloidal particles.) The chemical potential gradient forces are not all independent, however, as the Gibbs-Duhem equation at constant pressure and temperature requires

$$
\sum_{k} n_{k} \nabla \mu_{k}=0,
$$

where the sum is over all species, including the solvent; $n_{k}$ is the number density of species $k$. Thus, the average chemical potential gradient force exerted on a volume element of the suspension containing many particles is zero as it should be. This is why no chemical potential gradient force appears in the momentum balance for the solution in the continuum treatment of $\S 3$ (see (3.5)), nor in its suspension counterpart (4.32).

However, at the colloidal level the average motion of the suspension is not needed, which is the purpose of the momentum balance. Of interest is the motion of the various species relative to the solvent. Now, as shown by Batchelor (1976), a uniform force $\nabla \mu_{0} / v_{0}$, where the subscript 0 denotes the solvent and $v_{0}$ is the volume of a solvent molecule, exerted on both the solvent molecules and the colloidal particles produces no relative motion of colloidal particles and solvent and therefore the relative motion will be the same as if each colloidal particle is acted on by the steady applied force

$$
\boldsymbol{F}_{k}^{*}=\boldsymbol{F}_{k}+\frac{v_{k}}{1-\phi} \sum_{l=1}^{m} n_{l} \boldsymbol{F}_{l},
$$


and the solvent or fluid is force-free ( $l=0$ is excluded from the sum), and where

$$
\boldsymbol{F}_{k}=-\nabla \mu_{k}=-\sum_{l=1}^{m}\left(\frac{\partial \mu_{k}}{\partial n_{l}}\right)_{p . T} \nabla n_{l} .
$$

Here, $\phi=\sum_{l=1}^{m} n_{l} v_{l}=\sum_{l=1}^{m} \phi_{l}$ is the volume fraction of particles, with $v_{l}$ the volume of a particle of species $l$, and we have supposed that there are $m=k-1$ different colloidal particle species. Motion of particles due to a steady applied force in a fluid that is force-free is the same as sedimentation of the particles due to gravity.

The same result was obtained by Berne \& Pecora (1976, chap. 13) from a non-equilibrium thermodynamics approach when considering, as did Batchelor, multicomponent diffusion of colloidal particles in a solvent. This connection to multicomponent diffusion reveals the common underlying physical processes in the diffusiophoresis of a colloidal particle and the flux of one species driven by concentration gradient of another.

We now apply this result to our case of a single phoretic particle, which we shall label as particle $i$, in the presence of a concentration gradient of solute particles, labelled $j$. The mixture of solvent, solute and phoretic particle is incompressible and both the phoretic particle and the solute are large compared to the size of the solvent molecules. Under these conditions the chemical potential gradient forces are to leading order in the concentration of solute (Batchelor 1983)

$$
\begin{aligned}
& \boldsymbol{F}_{i}=-\frac{\beta}{8}(1+\lambda)^{3} v_{i} k T \nabla n_{j}+k T v_{i} \nabla n_{j}+O\left(k T \phi \nabla \phi_{j}\right), \\
& \boldsymbol{F}_{j}=-k T \nabla \ln n_{j}-k T v_{j} \beta_{j j} \nabla n_{j}+k T v_{j} \nabla n_{j}+O\left(k T \phi \nabla \phi_{j}\right),
\end{aligned}
$$

where $k T$ is the thermal energy and $\beta$ is the second virial coefficient

$$
\beta=8+3 \int_{2}^{\infty}\left[1-\mathrm{e}^{-\hat{\Phi}_{i j}}\right] s^{2} \mathrm{~d} s,
$$

with $\hat{\Phi}_{i j}(s)$ the $i-j$ the interparticle potential normalized by $k T$ and assumed here to be spherically symmetric. The variable $s$ is the separation distance between the centres of the spherical particles $i$ and $j$ non-dimensionalized by the sum of their radii, $a_{i}+a_{j}$ (see (4.21)). This interparticle potential $\hat{\Phi}_{i j}$ is the same as the interactive potential $\hat{V}$ in the continuum development of $\S 3$. The coefficient $\beta_{j j}$ in (4.5) is the same as (4.6) except for solute-solute interactive potentials $\hat{\Phi}_{j j}$.

The chemical potential gradient force on the solvent follows from the Gibbs-Duhem equation (4.1)

$$
-\nabla \mu_{0}=+\frac{1}{n_{0}}\left(k T(1-\phi) \nabla n_{j}+k T \phi_{j} \beta_{j j} \nabla n_{j}+k T \phi_{i} \frac{\beta}{8}(1+\lambda)^{3} k T \nabla n_{j}+\cdots\right) .
$$

For motion relative to the solvent such that there is no net chemical potential gradient force per unit volume on the solvent, it is the effective chemical potential gradient forces that are needed, which, from (4.2) to leading order in the concentration of solute, are:

$$
\begin{aligned}
\boldsymbol{F}_{i}^{*} & =-\frac{\beta}{8}(1+\lambda)^{3} v_{i} k T \nabla n_{j}+O\left(k T \phi \nabla \phi_{j}\right), \\
\boldsymbol{F}_{j}^{*} & =-k T \nabla \ln n_{j}-k T \beta_{j j} v_{j} \nabla n_{j}+O\left(k T \phi \nabla \phi_{j}\right) .
\end{aligned}
$$

Both the actual and the effective chemical gradient forces have been presented so that one can see clearly that the positive chemical potential gradient force on the 
solvent, $+k T(1-\phi) / n_{0} \nabla n_{j}$, which drives solvent motion opposite to that of the solute, cancels the positive potential gradient forces on the solute, $+k T v_{j} \nabla n_{j}$, and on the phoretic particle, $+k T v_{i} \nabla n_{j}$.

The resulting effective chemical potential gradient driving forces have the expected form. The force on a $j$ particle is simply minus the gradient of its chemical potential for dilute solutions the chemical potential is that of an ideal gas of $j$ particles, $\mu_{j}=k T \ln n_{j}$; the force is purely entropic. The force on the $i$ particle due to a concentration gradient of $j$ particles is seen to be proportional to the volume excluded to the centres of the of the $j$ particles, $(1+\lambda)^{3} v_{i}$, times the entropic gradient $-k T \nabla n_{j}$. For large $i$ particles this force can be very large, arising from the large entropic penalty associated with excluding the $j$ particles from the $i$-particle volume.

This distinction between the actual and the effective chemical potential gradient forces driving the motion is important. Consider first the case of no phoretic particle (no $i$ species) and very dilute solute so that the solute-solute interactive term $\beta_{j j} v_{j}$, which is $O\left(\phi_{j}\right)$ compared to the ideal gas chemical potential gradient term, is not important. Balancing the Stokes drag against the chemical potential gradient force gives a solute particle velocity $\boldsymbol{U}_{j}=-k T / 6 \pi \eta a_{j} \times \nabla \ln n_{j}$ and thus the wellknown expression for the diffusive flux of dilute solute: $\boldsymbol{j}^{\text {diff }}=n_{j} \boldsymbol{U}_{j}=-D_{j} \nabla n_{j}$, with $D_{j}=k T / 6 \pi \eta a_{j}$ the diffusivity of an isolated solute particle. (This is the same diffusive flux as used in the continuum treatment; see (3.4).) Whether one used that actual or the effective chemical potential gradient force does not matter for dilute solute as there is negligible flux of solvent. For a non-dilute solute, ignoring the flux of solvent and using the actual chemical potential gradient force would give a driving force proportional to $\beta_{j j}-1$, compared with $\beta_{j j}$ when the effective force is used, the latter, of course, being correct. The flux of non-dilute solute will drive a compensating flux of solvent in the opposite direction, necessitating the use of the effective chemical potential gradient forces for motion relative to zero-volume-flux axes. However, the velocity of the non-dilute solute is not simply the Stokes mobility times the driving force. The mobility will of course change from the isolated particle value, $1 / 6 \pi \eta a_{j}$, owing to hydrodynamic interactions between solute particles. But more critically, long-range hydrodynamic interactions among the solute particles must be summed properly to give the reduction in the velocity (or mobility) of the solute, and this leads directly to the problem of sedimentation.

When we consider the motion of the phoretic particle, this distinction between actual and effective forces becomes even more pronounced. In the absence of specific interparticle interactions between the phoretic particle and the solute, $\hat{\Phi}_{i j}=0, \beta=8$ and the actual chemical potential gradient force behaves as $\boldsymbol{F}_{i} \sim-3 \lambda v_{i} k T \nabla n_{j}$ and thus vanishes linearly in $\lambda$ as $\lambda \rightarrow 0$. In contrast, the effective chemical potential gradient force remains finite: $\boldsymbol{F}_{i}^{*} \sim-v_{i} k T \nabla n_{j}$. While it may seem intuitive that the force should vanish for very small solute particles, what is important is the vanishing of the motion of the phoretic particle, not necessarily the force (see $\S 4.2$ ). Because all the solute particles are 'settling' due to the chemical potential gradient forces acting on them - and note that the distinction between actual and effective forces on the solute does not matter to leading order in solute concentration - their long-range hydrodynamic interactions generate a back-flow of solvent that cancels the motion of the phoretic particle due to the effective force acting on it. As shown in $\S 4.4 .1$ the motion of the phoretic particle under the action of the effective chemical potential gradient forces vanishes as $\boldsymbol{U}_{i} \sim-\lambda^{2}\left(a^{2} / \eta\right) k T \nabla n_{j}$ as $\lambda \rightarrow 0$. If the actual chemical potential gradient force were used, the phoretic particle's velocity would not 
vanish; rather, the particle would move opposite to the driving force with velocity $\boldsymbol{U}_{i} \sim+\left(a^{2} / \eta\right) k T \nabla n_{j}$, which is clearly unphysical.

This rather subtle but important issue of the appropriate thermodynamic driving forces to use demonstrates why considerable care must be exercised in order to properly assess the effects of both thermodynamics and hydrodynamics on colloidal particle motion. And it shows the strength of the approach of Batchelor in separating the problem into one where the proper effective thermodynamic forces can be determined solely from statistical thermodynamic considerations, and then the hydrodynamic effects can be treated from a consideration of low-Reynolds-number flows that are independent of the origin of the driving forces.

We close this subsection with a discussion of the applicability of chemical potential gradient forces at the colloidal level. The chemical potential gradient or 'thermodynamic' driving forces are statistical in nature and one may question how they apply at the colloidal level when the dynamics of the individual colloidal particles are considered. Or viewed somewhat differently, why isn't the random Brownain motion of the colloidal particles sufficient? Imagine doing a dynamic simulation of interacting colloidal particles, for example by Stokesian dynamics. There would be no need to impose any chemical potential gradient forces; the thermal forces in the solvent that give rise to Brownian motion would be sufficient. If there was an initial concentration gradient of solute particles, then, just from the statistical random walk of the Brownian particles, as time evolved there would be a flux of solute down its concentration gradient until the concentration was uniform and equilibrium re-established. And there would be a compensating flux of solvent in the opposite direction so that the net flux of material (of volume) is zero, as this would be the appropriate reference frame for the motion. A phoretic particle immersed in this system would in general move both due to its own random walk and due to interactions with solute particles as they flowed down their concentration gradient and with the solvent as it flowed back up. These interactions are contained in the hydrodynamic mobility tensor $\boldsymbol{M}_{i j}$; the random thermal motions of the colloidal particles are coupled via hydrodynamics, which are long-ranged and must be summed properly. And it should be noted that in Stokesian dynamics the total force acting on any particle is identically zero at each and every instant; the random thermal forces are balanced by the hydrodynamic drag.

To obtain a steady process, one could imagine adding and removing solute particles at the boundaries so that the concentration gradient is maintained. Or one could repeat the transient simulation many times to have an ensemble of such systems. The average behaviour of the transient system, e.g. at the initial time when the solute gradient is uniform throughout the system, or the average behaviour of the steady-state system, would be the same as if there were steady effective chemical potential gradient forces acting on the colloidal particles and the solvent was force-free (the zero-volume-flux reference frame). It is this average motion that one wants to describe and it is in this sense that the chemical potential gradient forces and the probability distribution of the solute relative to the phoretic particle are to be understood.

\subsection{Convergent hydrodynamic interactions: back-flow}

Since the motion of the colloidal particles relative to the solvent resulting from the chemical potential gradient of the solute is the same as if there were steady applied forces on the particles and the solvent was force-free, the problem now becomes a hydrodynamic one of sedimentation of particles in a fluid. The long-range nature of hydrodynamic interactions in low-Reynolds-number or Stokes flow requires care 
when considering the motion of a particle due to interactions with a large collection of other particles. Specifically, the velocity disturbance due to a point force in a fluid decays as $1 / r$, and thus summing the pair-wise effects of particles subject to the same external force results in a badly divergent integral: $u \sim \int(1 / r) n \mathrm{~d} V \sim n R^{2}$, where $R$ is the size of the collection of particles with number density $n$. It is now well understood how to sum properly these long-range interactions so as to achieve absolutely convergent expressions. There are a number of different approaches (all resulting in the same expressions) and we give here a brief derivation that is especially transparent and can be used in a number of situations. The approach is given in detail in Brady et al. (1988) and forms the basis for Stokesian dynamics simulations of infinite suspensions of hydrodynamically interacting particles (Brady \& Bossis 1988).

We consider $N$ rigid particles dispersed in a fluid in volume $V$ in the thermodynamic limit: $N \rightarrow \infty, V \rightarrow \infty$ with $n=N / V$ fixed. Using the Green's function for Stokes flow of an incompressible Newtonian fluid, the velocity field $\boldsymbol{u}(\boldsymbol{x})$ at any point $\boldsymbol{x}$ in the fluid may be written without approximation as

$$
\boldsymbol{u}(\boldsymbol{x})=-\sum_{\alpha=1}^{N} \int_{S_{\alpha}} \boldsymbol{J}(\boldsymbol{x}-\boldsymbol{y}) \cdot \boldsymbol{\sigma}(\boldsymbol{y}) \cdot \boldsymbol{n} \mathrm{d} S_{y}-\int_{S_{\Gamma}^{\prime}}[\boldsymbol{J}(\boldsymbol{x}-\boldsymbol{y}) \cdot \boldsymbol{\sigma}(\boldsymbol{y}) \cdot \boldsymbol{n}+\boldsymbol{K}(\boldsymbol{x}-\boldsymbol{y}) \cdot \boldsymbol{u}(\boldsymbol{y}) \cdot \boldsymbol{n}] \mathrm{d} S_{y} .
$$

Here, $\boldsymbol{J}$ is the Green's function or Stokeslet

$$
\boldsymbol{J}(\boldsymbol{r})=\frac{1}{8 \pi \eta}\left(\frac{\boldsymbol{I}}{r}+\frac{\boldsymbol{r} \boldsymbol{r}}{r^{3}}\right),
$$

and

$$
\boldsymbol{K}(\boldsymbol{r})=-\frac{3}{4 \pi} \frac{\boldsymbol{r} \boldsymbol{r} \boldsymbol{r}}{r^{5}},
$$

corresponding to the stress field due to a point force. We note that $\boldsymbol{r}=\boldsymbol{x}-\boldsymbol{y}$, with $\boldsymbol{y}$ a point on the surface, and $\boldsymbol{n}(\boldsymbol{y})$ is the outer normal to the surfaces.

Equation (4.10) is an exact formulation for rigid particles. (Note that for rigid particles $\int_{S_{\alpha}} \boldsymbol{K} \cdot \boldsymbol{u} \cdot \boldsymbol{n} \mathrm{d} S_{y}=0$.) No divergences occur because we have a finite region bounded by the surface $S_{\Gamma}^{\prime}$. This is an arbitrary surface immersed in an unbounded statistically homogeneous suspension, i.e. the suspension continues outside $S_{\Gamma}^{\prime}$. If the radius of this surface is taken to be very large (with the origin located near the field point $\boldsymbol{x}$ ), the variation in $\boldsymbol{J}$ and $\boldsymbol{K}$ will be small over a surface element $\mathrm{d} S_{\Gamma}^{\prime}$ that passes through the fluid and around many particles. Thus, in the integrand of the second integral we may replace $\boldsymbol{\sigma}$ and $\boldsymbol{u}$ by averages. This is facilitated by first transforming from $S_{\Gamma}^{\prime}$ to a smooth macroscopic surface $S_{\Gamma}$ that cuts both fluid and particles; the averages thus formed are suspension averages - fluid and particle phase averages. Because the local normal to the surface $S_{\Gamma}^{\prime}$ varies on the particle scale, in addition to averages of $\boldsymbol{\sigma}$ and $\boldsymbol{u}$, the particles generate a quadrupolar contribution upon reduction from $S_{\Gamma}^{\prime}$ to $S_{\Gamma}$ (Brady et al. 1988).

At each point in the suspension, whether in the fluid or in a particle, Cauchy's equation of motion applies:

$$
\rho \frac{\mathrm{D} \boldsymbol{u}}{\mathrm{D} t}=\nabla \cdot \boldsymbol{\sigma}+\boldsymbol{f}
$$

where the 'body' force per unit volume $f$ is taken to be non-zero only within the particles. In the fluid the constitutive equation for the stress is that for a Newtonian fluid, $\boldsymbol{\sigma}=-p_{f} \boldsymbol{I}+2 \eta \boldsymbol{e}$, with $\mathbf{e}$ the rate of stain tensor, while in the particles the form of the constitutive relation is not known, but, fortunately, it is not needed. 
Averaging the point-wise Cauchy equation over a volume containing many particles gives (neglecting inertia)

$$
\nabla \cdot\langle\boldsymbol{\sigma}\rangle=-n\langle\boldsymbol{F}\rangle
$$

where

$$
\langle\boldsymbol{F}\rangle \equiv \frac{1}{N} \sum_{\alpha} \int_{V_{\alpha}} \boldsymbol{f}_{\alpha} \mathrm{d} V
$$

is the average non-hydrodynamic force acting on the particles, which equals the net force the particles exert on the fluid. Here, the angle brackets denote a volume average: $\langle\boldsymbol{\sigma}\rangle=(1 / V) \int \boldsymbol{\sigma} \mathrm{d} V$, which is equivalent to a surface average or an ensemble average for a statistically homogeneous suspension.

As shown by Brady et al. (1988), for spherical particles of radii $a$, (4.10) becomes

$$
\boldsymbol{u}(\boldsymbol{x})-\langle\boldsymbol{u}(\boldsymbol{x})\rangle=-\sum_{\alpha=1}^{N} \int_{S_{\alpha}} \boldsymbol{J} \cdot \boldsymbol{\sigma} \cdot \boldsymbol{n} \mathrm{d} S_{y}-\int_{V}\left(1+\frac{a^{2}}{6} \nabla^{2}\right) \boldsymbol{J} \cdot n\langle\boldsymbol{F}\rangle \mathrm{d} V+\frac{a^{2}}{9 \eta} n\langle\boldsymbol{F}\rangle,
$$

where the integral is over the entire volume surrounding the field point $\boldsymbol{x}$ and the sum is over all particles within the volume. The last two terms in (4.16) represent the 'back-flow' of fluid driven by the average pressure gradient that supports the 'weight' of the particles, $\nabla\langle p\rangle=n\langle\boldsymbol{F}\rangle$, where $\langle p\rangle=-(1 / 3) \boldsymbol{I}:\langle\boldsymbol{\sigma}\rangle$. And note that the appropriate frame of reference for the velocity is the volume-averaged velocity of the entire suspension - particles plus fluid: $\langle\boldsymbol{u}\rangle=(1 / V) \int \boldsymbol{u} \mathrm{d} V$. This expression for the fluid velocity is now absolutely convergent - the sum over the individual particles will exactly cancel the integral at large separations from the field point.

In order to determine the velocity of an individual particle in the suspension, we make use of Faxen's law for a spherical particle, which states

$$
\boldsymbol{U}_{\alpha}=\frac{\boldsymbol{F}_{\alpha}}{6 \pi \eta a_{\alpha}}+\left(1+\frac{a_{\alpha}^{2}}{6} \nabla^{2}\right) \boldsymbol{u}^{\prime}\left(\boldsymbol{x}_{\alpha}\right)
$$

where $\boldsymbol{u}^{\prime}$ is the velocity caused by all the other particles and the back-flow evaluated at the centre of particle $\alpha$. Returning to the exact integral representation for the solution (4.10) to obtain a convergent expression for $\nabla^{2} \boldsymbol{u}^{\prime}$, the velocity of any particle $\alpha$ becomes

$$
\boldsymbol{U}_{\alpha}-\langle\boldsymbol{u}\rangle=\sum_{\beta} \boldsymbol{M}_{\alpha \beta} \cdot \boldsymbol{F}_{\beta}-\int_{V}\left(1+\frac{a_{\alpha}^{2}+a^{2}}{6} \nabla^{2}\right) \boldsymbol{J} \cdot n\langle\boldsymbol{F}\rangle \mathrm{d} V+\left(\frac{a_{\alpha}^{2}+a^{2}}{9 \eta}\right) n\langle\boldsymbol{F}\rangle,
$$

where we have introduced the hydrodynamic mobility tensor $\boldsymbol{M}_{\alpha \beta}$ giving the velocity of particle $\alpha$ due to a force exerted on particle $\beta$. This expression forms the starting point for considering the motion of particles in a suspension. (Here we have just focused on the velocity-force relationship as that is all we shall need in this paper. Similar convergent expressions can be obtained for the rotational velocity, the torque, etc. See Brady et al. 1988 for details.)

To apply this to the problem at hand of a large particle in a dispersion of solute particles, we label the large particle as ' $i$ ' and the solute particles as ' $j$ ' to keep with the notation used by Batchelor. Further, we are interested in a single large particle and thus the number density and average force in the back-flow integral come from the solute particles. Finally, we want to average over the distribution of identical 
solute particles. The sum in (4.18) becomes an integral with the probability weight and the average velocity of the large particle becomes (Batchelor 1982)

$$
\begin{aligned}
\left\langle\boldsymbol{U}_{i}\right\rangle-\langle\boldsymbol{u}\rangle & =\frac{\boldsymbol{F}_{i}}{6 \pi \eta a_{i}}+\int_{r>a_{i}+a_{j}}\left\{\left[\left(\boldsymbol{M}_{i i}-\tilde{\boldsymbol{I}}_{i i}\right) \cdot \boldsymbol{F}_{i}+\boldsymbol{M}_{i j} \cdot \boldsymbol{F}_{j}\right] n_{j} p_{i j}\right. \\
- & \left.\left(1+\frac{a_{i}^{2}+a_{j}^{2}}{6} \nabla^{2}\right) \boldsymbol{J} \cdot n_{j}\left\langle\boldsymbol{F}_{j}\right\rangle\right\} \mathrm{d} \boldsymbol{r}-\frac{2}{9} \frac{a_{i}^{2}}{\eta} n_{j}\left\langle\boldsymbol{F}_{j}\right\rangle\left(1+3 \lambda+\lambda^{2}\right),
\end{aligned}
$$

where, $n_{j} p_{i j}(\boldsymbol{r})$ is the probability density for finding a solute particle $j$ relative to the particle $i, \boldsymbol{r}=\boldsymbol{x}_{j}-\boldsymbol{x}_{i}$ is the relative separation vector, $\tilde{\boldsymbol{I}}_{i i}=\boldsymbol{I} / 6 \pi \eta a_{i}$, and the last term on the right-hand side of (4.19) comes from the last term in (4.18) and the back-flow integral from 0 to $a_{i}+a_{j}$. The size ratio $\lambda$ is

$$
\lambda \equiv \frac{a_{j}}{a_{i}}
$$

Following Batchelor, we non-dimensionalize with

$$
\boldsymbol{M}_{i j}=\frac{1}{3 \pi \eta\left(a_{i}+a_{j}\right)} \hat{\boldsymbol{M}}_{i j}, \quad s=\frac{2 r}{\left(a_{i}+a_{j}\right)},
$$

and (4.19) becomes

$$
\begin{aligned}
\left\langle\boldsymbol{U}_{i}\right\rangle-\langle\boldsymbol{u}\rangle= & \frac{\boldsymbol{F}_{i}}{6 \pi \eta a_{i}}-\frac{2}{9} \frac{a_{i}^{2}}{\eta} n_{j} \boldsymbol{F}_{j}\left(1+3 \lambda+\lambda^{2}\right) \\
& +\frac{a_{i}^{2}}{\eta} \frac{1}{6 \pi}\left(\frac{1+\lambda}{2}\right)^{3} \int_{s \geqslant 2}\left(\hat{\boldsymbol{M}}_{i i}-\hat{\boldsymbol{I}}_{i i}\right) \cdot \boldsymbol{F}_{i} n_{j} p_{i j} \mathrm{~d} \boldsymbol{s} \\
& +\frac{a_{i}^{2}}{\eta} \frac{1}{6 \pi}\left(\frac{1+\lambda}{2}\right)^{2} \int_{s \geqslant 2}\left[\hat{\boldsymbol{M}}_{i j} p_{i j}-\frac{3}{4}\left(1+\gamma \nabla^{2}\right) \hat{\boldsymbol{J}}\right] \cdot n_{j} \boldsymbol{F}_{j} \mathrm{~d} \boldsymbol{s},
\end{aligned}
$$

where $\gamma=(2 / 3)\left(1+\lambda^{2}\right) /(1+\lambda)^{2}$, and we have assumed that all $j$ particles are identical so that the force on an individual $j$ particle is equal to the average $\boldsymbol{F}_{j}=\left\langle\boldsymbol{F}_{j}\right\rangle$.

Each term in the expression for the average velocity of the $i$ th particle has a distinct physical interpretation. The first term on the right-hand side of (4.22) is simply the Stokes velocity of an isolated $i$ particle subject to an external force $\boldsymbol{F}_{i}$. The second term is a reduction (assuming $\boldsymbol{F}_{i}$ and $\boldsymbol{F}_{j}$ point in the same direction) in the $i$-particle velocity due to the back-flow arising from the volume $\left(r<a_{i}+a_{j}\right)$ excluded to the centre of a $j$ particle. The third term is the change in the mobility of the $i$ particle owing to hydrodynamic interactions with $j$ particles distributed with probability density $n_{j} p_{i j}$; since $\left(\hat{\boldsymbol{M}}_{i i}-\hat{\boldsymbol{I}}_{i i}\right)$ decays as $1 / s^{4}$ the integral is absolutely convergent. The final term is the difference between the enhanced fall speed of the $i$ particle caused by the force exerted on the $j$ particle distributed according to $p_{i j}, \hat{\boldsymbol{M}}_{i j} p_{i j}$, and the back-flow associated with the uniform distribution of $j$ particles; at large separations $\hat{\boldsymbol{M}}_{i j}-(3 / 4)\left(1+\gamma \nabla^{2}\right) \hat{\boldsymbol{J}} \sim 1 / s^{7}$ and the integral is absolutely convergent.

Equation (4.22) applies for any size ratio $\lambda$, for any distribution $p_{i j}$ of solute particles relative to particle $i$, and for any constant external forces exerted on particles $i$ or $j$. The only assumption of diluteness we have made is in using the pair-wise mobility tensors, $\hat{\boldsymbol{M}}_{i j}(\boldsymbol{s})$, rather than the many-body tensors needed at high $j$-particle concentration. But this does not change the structure of the problem nor the main conclusions reached below.

Equation (4.22) applies to the familiar example of all particles being identical $(i=j)$ and falling due to gravity $\boldsymbol{F}_{i}=\boldsymbol{F}_{j}=\boldsymbol{F}^{g}=\Delta \rho \boldsymbol{g} 4 \pi a^{3} / 3$. If we take the distribution to be 
uniform outside the excluded volume, $p_{i j}=1$, then we recover Batchelor's expression (Batchelor 1972) for the reduction in the sedimentation velocity of a particle in a suspension:

$$
\left\langle\boldsymbol{U}^{g}\right\rangle=\frac{\boldsymbol{F}^{g}}{6 \pi \eta a}(1-6.55 \phi),
$$

where $\phi=n 4 \pi a^{3} / 3$ is the volume fraction of the suspended particles.

Equation (4.22) also applies to the case where there is no force on the $i$ particle but all the $j$ particles are falling due to gravity. If we again take the distribution of $j$ particles to be uniform outside the excluded volume, $p_{i j}=1$, only the back-flow contribution from the excluded volume remains and the $i$ particle velocity becomes

$$
\left\langle\boldsymbol{U}_{i}\right\rangle=-\frac{2}{9} \frac{a_{i}^{2}}{\eta} \phi_{j}\left(\rho_{j}-\rho_{f}\right) \boldsymbol{g} \times\left(1+3 \lambda+\lambda^{2}\right),
$$

where $\rho_{j}$ is the density of a $j$ particle. Although there is no force on the $i$ particle it moves nonetheless, rising with the back-flow of fluid. The same result can be obtained from a simple macroscopic continuum balance. While the $i$ particle has no density difference with the fluid $\left(\rho_{i}=\rho_{f}\right)$, it is less dense than the suspension and thus will rise due to buoyancy. Treating the suspension of $j$ particles as a continuum, the suspension density is $\rho_{\text {sus }}=\rho_{f}\left(1-\phi_{j}\right)+\rho_{j} \phi_{j}$, and thus the buoyant force exerted on the $i$ particle is $\left(4 \pi a_{i}^{3} / 3\right) \times\left(\rho_{f}-\rho_{\text {sus }}\right) \boldsymbol{g}=-\left(4 \pi a_{i}^{3} / 3\right)\left(\rho_{j}-\rho_{f}\right) \phi_{j} \boldsymbol{g}$. Balancing this buoyant force with the Stokes drag gives (4.24) without the finite-size factor $\left(1+3 \lambda+\lambda^{2}\right)$, which cannot be obtained from the continuum-level description. This simple and obvious result should reassure the reader that the colloidal treatment can indeed recover (and extend) the macroscopic continuum approach. It also shows clearly that what is a force at one level is not necessarily a force at a different level - there is no force on the $i$ particle at the colloidal level, while the same particle viewed at the continuum level has a buoyant force - and yet the two approaches describe the same physics (in the limit of small $\lambda$ ).

\subsection{Connection between 'suspension' and 'solution' properties}

Before proceeding further, it may be useful to make precise the connection between the average suspension stress, $\langle\boldsymbol{\Sigma}\rangle$, and average suspension velocity, $\langle\boldsymbol{u}\rangle$, and what was called the solution stress, $\boldsymbol{\Sigma}$, and solution velocity, $\boldsymbol{v}$, in the continuum development of $\S 3$. A continuum 'point' corresponds to a volume element of the suspension that contains many solute particles and so the connection is to average suspension properties. Further, the phoretic particle in the continuum approach must be viewed as an object much larger than any continuum point (see the discussion in $\S 6$ ) and therefore the colloidal particle $i$ should not be included in the suspension average. Of course, the whole point of the colloidal perspective is to remove this restriction, but nevertheless the colloidal approach can produce the continuum description.

In the colloidal treatment the volume average suspension velocity is given by

$$
\langle\boldsymbol{u}\rangle=(1-\phi)\left\langle\boldsymbol{u}_{f}\right\rangle+\phi\left\langle\boldsymbol{u}_{p}\right\rangle,
$$

where $\phi$ is the volume fraction of particles in the suspension and $\left\langle\boldsymbol{u}_{f}\right\rangle=$ $\left(1 / V_{f}\right) \int_{V_{f}} \boldsymbol{u} \mathrm{d} V$ and $\left\langle\boldsymbol{u}_{p}\right\rangle=\left(1 / V_{p}\right) \int_{V_{p}} \boldsymbol{u} \mathrm{d} V$ are the volume average velocities of the fluid and particles phases, with $V_{f}$ and $V_{p}$ being their respective volumes. This volume average suspension velocity is what was called the 'solution' velocity $\boldsymbol{v}$ in the continuum treatment of $\S 3$, and thus we have the connection

$$
\boldsymbol{v} \equiv\langle\boldsymbol{u}\rangle .
$$


Of course for very dilute solute concentrations, $\phi=\phi_{s} \ll 1$, the solution velocity is the same as the fluid or solvent velocity: $\boldsymbol{v}=\left\langle\boldsymbol{u}_{f}\right\rangle=\boldsymbol{u}$. Note that since both the fluid and the particles are incompressible, the volume average velocity is also incompressible:

$$
\nabla \cdot\langle\boldsymbol{u}\rangle=0 .
$$

A similar relation exists for the solution stress $\boldsymbol{\Sigma}$ introduced in $\S 3$, although the situation is a bit more involved because there are contributions to the average stress in a suspension in addition to the average of the Cauchy or hydrodynamic stress $\langle\boldsymbol{\sigma}\rangle$. These additional stresses arise from two sources. First, if there are interparticle forces between the colloidal particles, e.g. van der Waals interactions or perhaps a spring-like bond as in bead-spring models of polymer solutions, then there is a contribution to the suspension stress $n\left\langle\boldsymbol{S}^{I}\right\rangle=-n\left\langle\boldsymbol{X} \boldsymbol{F}^{I}\right\rangle$, where $\boldsymbol{X}$ denotes the vector of positions of all the particles and $\boldsymbol{F}^{I}$ the vector of interparticle forces. This contribution is of the same form as in atomic or molecular systems, and since it involves interactions between pairs of particles it is $O\left(n^{2}\right)$. The second contribution arises from the thermal fluctuations in the solvent that give rise to the Brownian motion of the colloidal particles. There are hydrodynamical flows resulting from the Brownian forces on the colloidal particles that generate a Brownian stresslet $\boldsymbol{S}^{B}$ (see Brady 1993) and thus a contribution to the suspension stress $n\left\langle\boldsymbol{S}^{B}\right\rangle$. Like the interparticle force contribution, the Brownian stresslet requires the interactions of at least a pair of particles (for spherical particles) and therefore its contribution is also $O\left(n^{2}\right)$. Even for an isolated Brownian particle, however, the Maxwell-Boltzmann distribution of random thermal velocities generates an ideal gas contribution, $-n k T I$, to the average stress. The average of the Cauchy or hydrodynamic stress can be written as

$$
\langle\boldsymbol{\sigma}\rangle=-(1-\phi)\left\langle p_{f}\right\rangle \boldsymbol{I}+2 \eta\langle\mathbf{e}\rangle+n\left\langle\boldsymbol{S}^{H}\right\rangle,
$$

where $\left\langle p_{f}\right\rangle=\left(1 / V_{f}\right) \int_{V_{f}} p_{f} \mathrm{~d} V$ is the average of the pressure in the fluid and $\mathbf{S}^{H}$ is the hydrodynamic stresslet (see Batchelor 1970). Thus, the average stress in a suspension is

$$
\langle\boldsymbol{\Sigma}\rangle=-\left[(1-\phi)\left\langle p_{f}\right\rangle+n k T\right] \boldsymbol{I}+2 \eta\langle\mathbf{e}\rangle+n\left\{\left\langle\boldsymbol{S}^{H}\right\rangle+\left\langle\boldsymbol{S}^{I}\right\rangle+\left\langle\boldsymbol{S}^{B}\right\rangle\right\} .
$$

And we can now identify the solution stress of $\S 3, \boldsymbol{\Sigma}$, with the suspension stress, $\langle\boldsymbol{\Sigma}\rangle$ :

$$
\boldsymbol{\Sigma} \equiv\langle\boldsymbol{\Sigma}\rangle \text {. }
$$

(The reader should not confuse the use of the same symbol $\Sigma$ in the two contexts above.)

Both the interparticle and Brownian stresslets are $O(n)$ and thus contribute $O\left(n^{2}\right)$ to the suspension stress. However, an individual spherical particle has a hydrodynamic stresslet of $20 \pi a^{3} \eta\langle\boldsymbol{e}\rangle / 3$, which gives the familiar Einstein correction to the solvent viscosity $5 \phi / 2$. Thus, correct to $O\left(n^{2}\right)$ the solution stress is

$$
\boldsymbol{\Sigma}=-\left[(1-\phi)\left\langle p_{f}\right\rangle+n k T\right] \boldsymbol{I}+2 \eta\left(1+\frac{5}{2} \phi\right)\langle\mathbf{e}\rangle .
$$

In the continuum development of $\S 3$ (see (3.1) and (3.2)) we neglected the Einstein viscosity correction to the solvent viscosity, but kept the solute contribution to the pressure. While both are of first order in the solute concentration, they are of different character: the Einstein correction to the viscosity is proportional to the volume fraction of the solute, while the solute pressure is not and thus remains even as $\phi \rightarrow 0$. It should be recalled, however, that because of the incompressibility of the solution, $\nabla \cdot \boldsymbol{v}=0$ (or of the suspension $\nabla \cdot\langle\boldsymbol{u}\rangle=0$ ), the average pressure of the fluid, $(1-\phi)\left\langle p_{f}\right\rangle$, will take on whatever form is necessary so that the total pressure, 
$p=(1-\phi)\left\langle p_{f}\right\rangle+n k T$, ensures incompressibility. (Note that in $\S 3$ what was called the fluid pressure, $p_{f}$, is formally $p_{f}=(1-\phi)\left\langle p_{f}\right\rangle$; the factor of $(1-\phi)$ arising simply from whether one averages over the total volume, $V$, or just the fluid volume, $V_{f}$. This distinction does not matter because of incompressibility.)

And finally, the interactive force of the solute particle with the phoretic particle in the continuum treatment is the same as the interparticle colloidal force defined in (4.6): $\boldsymbol{f}_{s}=-\nabla V=-\nabla \Phi_{i j}$. This (average) solute 'body' force is then to be used with the equation for conservation of momentum

$$
0=\nabla \cdot\langle\boldsymbol{\Sigma}\rangle+n f_{s},
$$

where we have neglected the inertial terms on the left-hand side for low-Reynoldsnumber flows. (Averaging the acceleration of the suspension will generate additional 'Reynolds' stress contributions to the average stress.) Note that the chemical potential gradient forces do not appear in the momentum balance (4.32) because the volume element also includes the solvent and thus the Gibbs-Duhem equation (4.1) applies.

In $\S 6$, we show the appropriate forms for the suspension stress and solute flux to include solute-solute interactions in the continuum approach to phoretic motion.

It should be recalled, however, that at the colloidal level, down amongst the colloidal particles, one does not need to determine the average suspension velocity, $\langle\boldsymbol{u}\rangle$, nor the average suspension stress, $\langle\boldsymbol{\Sigma}\rangle$. What is of interest is the motion of the colloidal particles relative to the average velocity, which would also be the motion relative to the laboratory frame.

\subsection{Direct contribution from the chemical potential gradient and back-flow}

We now use the effective chemical potential gradient forces, (4.8)-(4.9), in (4.22) to get the mean velocity of the $i$ particle due to the constant concentration gradient of $j$ particles. To do so we need the pair-distribution function $p_{i j}$. As discussed below (\$4.5), to leading order in the weak concentration gradient $\nabla n_{j}$, in (4.22) we need only the equilibrium pair-distribution function, which is given by the Boltzmann distribution: $p_{i j}^{e q}=\mathrm{e}^{-\hat{\phi}_{i j}}$. Thus, (4.22) becomes

$$
\begin{aligned}
\left\langle\boldsymbol{U}_{i}\right\rangle-\langle\boldsymbol{u}\rangle= & -\frac{2}{9} \frac{a_{i}^{2}}{\eta} k T \nabla n_{j} \frac{\beta}{8}(1+\lambda)^{3}+\frac{2}{9} \frac{a_{i}^{2}}{\eta} k T \nabla n_{j}\left(1+3 \lambda+\lambda^{2}\right) \\
& -\frac{2}{9} \frac{a_{i}^{2}}{\eta}\left(\frac{1+\lambda}{2}\right)^{3} \frac{\phi_{j}}{\lambda^{3}} k T \nabla n_{j} \cdot \int_{s \geqslant 2}\left(\hat{\boldsymbol{M}}_{i i}-\hat{\boldsymbol{I}}_{i i}\right) \mathrm{e}^{-\hat{\boldsymbol{\phi}}_{i j}} \mathrm{~d} \boldsymbol{s} \\
& -\frac{2}{9} \frac{a_{i}^{2}}{\eta}\left(\frac{1+\lambda}{2}\right)^{2} k T \nabla n_{j} \cdot \frac{3}{4 \pi} \int_{s \geqslant 2}\left[\hat{\boldsymbol{M}}_{i j} \mathrm{e}^{-\hat{\Phi}_{i j}}-\frac{3}{4}\left(1+\gamma \nabla^{2}\right) \hat{\boldsymbol{J}}\right] \mathrm{d} \boldsymbol{s},
\end{aligned}
$$

where we have used $v_{i} n_{j}=\phi_{j} / \lambda^{3}$. Equation (4.33) applies for any size ratio (the solute need not be smaller than the $i$ particle) and any interparticle potential $\hat{\Phi}_{i j}$. The only restriction is that the solute concentration is small, $\phi_{j} \ll 1$. (To leading order in the solute volume fraction the $\beta_{j j} v_{j}$ term in (4.9) is not needed.)

Our goal is to connect to the continuum description in $\S 3$ and thus we want to consider small solute, $\lambda \ll 1$, with or without an interparticle potential. It is most clear if we discuss the two cases, with/without interparticle potential, separately. 


\subsubsection{No interparticle potential}

For no potential interactions between the $i$ particle and the solute we have $\hat{\Phi}_{i j}=0$, and $\beta=8$. Thus, the first two terms on the right-hand side of (4.33) combine to give

$$
-\frac{2}{9} \frac{a_{i}^{2}}{\eta} k T \nabla n_{j}(1+\lambda)^{3}+\frac{2}{9} \frac{a_{i}^{2}}{\eta} k T \nabla n_{j}\left(1+3 \lambda+\lambda^{2}\right)=-\frac{2}{9} \frac{a_{i}^{2}}{\eta} k T \nabla n_{j}\left(2 \lambda^{2}+\lambda^{3}\right) .
$$

The (large) entropic driving force on particle $i$ is compensated by the back-flow of fluid (solvent) induced by the constant force on all the solute particles, $j$, so that the net velocity is proportional to $a_{j}^{2}$ rather than to $a_{i}^{2}$.

The third term on the right-hand side of (4.33), which corresponds to the change in mobility of particle $i$ due to hydrodynamic interactions with a particle $j$, is proportional to the volume fraction of $j$ particles and appears to diverge as $\lambda \rightarrow 0$. However, the change in mobility of the $i$ particle due to interaction with a $j$ particle is small when $\lambda$ is small. From the well-known formulae for the mobility tensors, $\hat{\boldsymbol{M}}_{i i}-\hat{\boldsymbol{I}}_{i i} \sim \lambda^{3} / s^{4}$, and the $\lambda$ cancel out. As shown by Batchelor (1982), in the small $\lambda$ limit the third term becomes

$$
-\frac{2}{9} \frac{a_{i}^{2}}{\eta}\left(\frac{1+\lambda}{2}\right)^{3} \frac{\phi_{j}}{\lambda^{3}} k T \nabla n_{j} \cdot \int_{s \geqslant 2}\left(\hat{\boldsymbol{M}}_{i i}-\hat{\boldsymbol{I}}_{i i}\right) \mathrm{d} \boldsymbol{s}=+\frac{2}{9} \frac{a_{i}^{2}}{\eta} k T \nabla n_{j} \frac{5}{2} \phi_{j}[1+O(\lambda)] .
$$

The physical interpretation is that the small $j$ particles change the viscosity of the solvent by the Einstein correction $\eta_{\text {eff }}=\eta\left(1+(5 / 2) \phi_{j}\right)$. Particle $i$ generates a disturbance flow that decays as $1 / s$, which induces a force dipole or hydrodynamic stresslet in particle $j$ of magnitude $\lambda^{3} / s^{2}$, which propagates a stresslet velocity disturbance, $\lambda^{3} / s^{2} \times 1 / s^{2} \sim \lambda^{3} / s^{4}$, back to particle $i$. It is this induced stresslet that gives the Einstein viscosity correction. However, note that this Einstein viscosity correction enters into the mobility reduction, but not the back-flow contribution.

The final contribution to the mean velocity of particle $i$ involves the integral of the difference between the mobility of particle $i$ due to a force on particle $j$ and the far-field form of this interaction embodied by $J$. From the well-known formulae for mobility interactions $\left(\hat{\boldsymbol{M}}_{i j}-(3 / 4)\left(1+\gamma \nabla^{2}\right) \hat{\boldsymbol{J}}\right) \sim \lambda^{3} / s^{7}$ and thus the last term is small:

$$
-\frac{2}{9} \frac{a_{i}^{2}}{\eta}\left(\frac{1+\lambda}{2}\right)^{2} k T \nabla n_{j} \cdot \frac{3}{4 \pi} \int_{s \geqslant 2}\left[\hat{\boldsymbol{M}}_{i j}-\frac{3}{4}\left(1+\gamma \nabla^{2}\right) \hat{\boldsymbol{J}}\right] \mathrm{d} \boldsymbol{s}=O\left(\frac{a_{i}^{2}}{\eta} k T \nabla n_{j} \lambda^{3}\right) .
$$

Bringing all the contributions together we have

$$
\left\langle\boldsymbol{U}_{i}\right\rangle-\langle\boldsymbol{u}\rangle=-\frac{2}{9} \frac{a_{i}^{2}}{\eta} k T \nabla n_{j}\left[2 \lambda^{2}-\frac{5}{2} \phi_{j}+O\left(\lambda^{3}, \phi_{j} \lambda\right)\right] .
$$

We are now in a position to make a connection to the continuum perspective. In the continuum limit, the solute has no size and thus the two limits $\lambda=0$ and $\phi_{j}=0$ hold, and we recover $\left\langle\boldsymbol{U}_{i}\right\rangle-\langle\boldsymbol{u}\rangle=0$ as expected (see $\S 3.1$ ): the solute is so dilute that it does not change the viscosity of the solution and the back-flow contribution exactly cancels the velocity due to the entropic force on the $i$ particle. It is reassuring that the colloidal and continuum perspectives give the same result of zero net motion when the $i$ particle is much larger than the solute and there is no specific interparticle interaction between the $i$ particle and the solute.

It is tempting to associate the $2 \lambda^{2}$ term in (4.37) with the phoretic velocity found in (3.35), identifying $a_{i}^{2} \lambda^{2}=a_{j}^{2}$ with $b^{2}$. After all, there are hard-sphere excluded-volume interactions between the $i$ particle and the solute for distances $r<\left(a_{i}+a_{j}\right)$ and the 
coefficient $4 / 9$ is not far from $1 / 2$. However, such an association is false. The only proper interpretation of the connection between (4.37) and the continuum limit is that they both give zero for the particle velocity in the limit $\lambda \rightarrow 0$.

\subsubsection{Interparticle interaction: hard-sphere at $s=s_{c}=2(1+\Delta)$}

We now consider there to be interparticle interactions between particle $i$ and the solute $j$. We shall take this interaction to have a range $\left(a_{i}+a_{j}\right) \Delta$, beyond which the interparticle potential is zero; in dimensionless form

$$
\hat{\Phi}_{i j}=0, \quad \text { for } s>2(1+\Delta) .
$$

Two modifications to the above analysis occur. The virial coefficient now becomes $\beta=8+3 \int_{2}^{2(1+\Delta)}\left[1-\mathrm{e}^{-\hat{\phi}_{i j}}\right] s^{2} \mathrm{~d} s$, and the last term on the right-hand side of (4.33) now has a contribution from 2 to $2(1+\Delta)$, where $\hat{\boldsymbol{M}}_{i j} \mathrm{e}^{-\hat{\Phi}_{i j}} \neq(3 / 4)\left(1+\gamma \nabla^{2}\right) \hat{\boldsymbol{J}}$ to $O\left(\lambda^{3}\right)$. Carrying out the angular integration for this last term and combining, the velocity of particle $i$ now becomes

$$
\begin{aligned}
\left\langle\boldsymbol{U}_{i}\right\rangle-\langle\boldsymbol{u}\rangle=-\frac{2}{9} \frac{a_{i}^{2}}{\eta} k T \nabla n_{j}\left[2 \lambda^{2}+\lambda^{3}\right. & +\frac{3}{8}(1+\lambda)^{3} \int_{2}^{2(1+\Delta)}\left[1-\mathrm{e}^{-\hat{\Phi}_{i j}}\right] \\
& \left.\times\left(s^{2}-\frac{2}{1+\lambda} s\right) \mathrm{d} s+O\left(\lambda^{3}, \phi_{j}\right)\right] .
\end{aligned}
$$

Note, at this point we have not yet specified the range $\Delta$ of the interparticle potential, nor its form. To connect to the continuum analysis in $\S 3.2$ we now take the potential to be hard-sphere: $\mathrm{e}^{-\hat{\phi}_{i j}}=0$ for $s \leqslant 2(1+\Delta)$, and $\left\langle\boldsymbol{U}_{i}\right\rangle$ becomes

$$
\left\langle\boldsymbol{U}_{i}\right\rangle-\langle\boldsymbol{u}\rangle=-\frac{2}{9} \frac{a_{i}^{2}}{\eta} k T \nabla n_{j}\left[\frac{3}{2} \Delta^{2}+\Delta^{3}+2 \lambda^{2}+O\left(\lambda \Delta, \lambda^{3}, \phi_{j}\right)\right] .
$$

Equation (4.40) applies for any range $\Delta$ (for small $\lambda$ ). In the limit $\Delta \rightarrow 0$, the potential contribution vanishes and (4.37) is recovered as it should be. To connect to the continuum limit, however, we take the limit $\lambda \rightarrow 0$ first, followed by $\Delta \rightarrow 0$ :

$$
\left\langle\boldsymbol{U}_{i}\right\rangle-\langle\boldsymbol{u}\rangle=-\frac{2}{9} \frac{a_{i}^{2}}{\eta} k T \nabla n_{j}\left[\frac{3}{2} \Delta^{2}+O\left(\Delta^{3}, \lambda \Delta, \lambda^{2}, \phi_{j}\right)\right] .
$$

It is now correct to identify the hard-sphere excluded-volume interaction length $b$ in the continuum treatment (3.35) as $\left(a_{i}+a_{j}\right) \Delta=b$ for small $\lambda$. Thus, (4.41) gives

$$
\left\langle\boldsymbol{U}_{i}\right\rangle-\langle\boldsymbol{u}\rangle=-\frac{1}{3} \frac{b^{2}}{\eta} k T \nabla n_{j},
$$

which compares well with (3.35) as far as the scalings are concerned; the numerical factor is $1 / 3$ rather than $1 / 2$, however.

One could ignore the numerical factor difference and have the opinion that the two approaches - continuum and colloidal - agree well enough. However, one should be able to recover the exact same result, not approximately the same. Further, we never had to determine the departure of the solute concentration distribution from equilibrium as we did in the continuum perspective in arriving at (3.17), and this distribution was important in obtaining the slip velocity and thus the phoretic velocity. The resolution of this apparent paradox is that there is an additional contribution to the motion of the $i$ particle that arises from Brownian motion and interparticle forces that must be considered. 


\subsection{Indirect contribution from Brownian and interparticle forces}

When colloidal particles exert a mutual interactive force on one another, e.g. $\boldsymbol{F}_{i}=-\boldsymbol{F}_{j}=\boldsymbol{F}_{i j}(\boldsymbol{r})$, this causes the particles to move relative to one another and contributes to the motion of the colloidal particle $i$. Whether this interactive force is due to an actual colloidal interparticle potential, $\boldsymbol{F}_{12}^{(I)}=-\nabla_{1} \Phi$, or due to the relative Brownian motion of the two particles, $\boldsymbol{F}_{12}^{(B)}=-k T \partial \ln P\left(\boldsymbol{x}_{1}, \boldsymbol{x}_{2}\right) / \partial \boldsymbol{x}_{1}=k T \nabla_{r} \ln g$, where $P\left(\boldsymbol{x}_{1}, \boldsymbol{x}_{2}\right)=n_{1} n_{2} g(\boldsymbol{r})$ is the joint probability density of their centres, does not matter. (Recall that at the Smoluchowski level of colloidal dynamics the statistical or 'thermodynamic' Brownian force appears as the gradient of the probability density.) The additional velocity of the $i$ th particle when averaged over the probability distribution of the $j$ particles is given by (Batchelor 1982)

$$
\left\langle\Delta \boldsymbol{U}_{i}\right\rangle=\frac{1}{4} k T\left(a_{i}+a_{j}\right)^{2} n_{j} \int_{s \geqslant 2}\left(\boldsymbol{M}_{i i}-\boldsymbol{M}_{i j}\right) \cdot \nabla\left(\hat{\Phi}_{i j}+\ln p_{i j}\right) p_{i j} \mathrm{~d} \boldsymbol{s} .
$$

In equilibrium, $\hat{\Phi}_{i j}+\ln p_{i j}=0$, and there is no net average velocity of the $i$ th (or any) particle; net motion is a result of the distribution being out of equilibrium. Here, the distribution is driven out of equilibrium by the chemical potential gradient of the solute. (Note that since the forces on particles $i$ and $j$ are equal and opposite, there is no net force exerted on an average volume element of the suspension.)

The pair-distribution function $p_{i j}$ satisfies the Smoluchowski equation

$$
\left.\begin{array}{l}
\frac{\partial p_{i j}}{\partial t}+\nabla \cdot \boldsymbol{w}_{i j} p_{i j}=\nabla \cdot \boldsymbol{D}_{i j} \cdot \nabla\left(\hat{\Phi}_{i j}+\ln p_{i j}\right) p_{i j}, \\
p_{i j} \sim 1, \quad \text { as } \quad r \rightarrow \infty, \\
\boldsymbol{n} \cdot \boldsymbol{D}_{i j} \cdot \nabla\left(\hat{\Phi}_{i j}+\ln p_{i j}\right) p_{i j}-\boldsymbol{n} \cdot \boldsymbol{w}_{i j} p_{i j}=0, \quad \text { at } \quad r=a_{i}+a_{j},
\end{array}\right\}
$$

where $\boldsymbol{w}_{i j}$ is the velocity of particle $j$ minus the velocity of particle $i$ arising from the chemical potential gradient of species $j$ and $\boldsymbol{n}$ is the normal from particle $i$ to $j$. The relative diffusivity of the pair is given by $\boldsymbol{D}_{i j}=k T\left(\boldsymbol{M}_{i i}+\boldsymbol{M}_{j j}-\boldsymbol{M}_{i j}-\boldsymbol{M}_{j i}\right)$.

The relative velocity due to the chemical potential gradient is given by

$$
\boldsymbol{w}_{i j}=\boldsymbol{U}_{j}-\boldsymbol{U}_{i}=\left(\boldsymbol{M}_{j j}-\boldsymbol{M}_{i j}\right) \cdot \boldsymbol{F}_{j}^{*}+\left(\boldsymbol{M}_{j i}-\boldsymbol{M}_{i i}\right) \cdot \boldsymbol{F}_{i}^{*},
$$

where $\boldsymbol{F}_{i}^{*}$ and $\boldsymbol{F}_{j}^{*}$ are given by (4.8) and (4.9), respectively. Noting that

$$
\boldsymbol{F}_{i}^{*}=v \boldsymbol{F}_{j}^{*}, \quad \text { with } \quad v=\frac{\beta}{8}(1+\lambda)^{3} \frac{\phi_{j}}{\lambda^{3}},
$$

the relative velocity can be written as

$$
\boldsymbol{w}_{i j}=\boldsymbol{w}_{i j}^{(0)} \cdot[\hat{L}(s) \hat{\boldsymbol{s}} \hat{\boldsymbol{s}}+\hat{M}(s)(\boldsymbol{I}-\hat{\boldsymbol{s}} \hat{\boldsymbol{s}})],
$$

where $\hat{s}$ is a unit vector in the direction of $s$, and we have introduced hydrodynamic mobility functions $\hat{L}(s)$ and $\hat{M}(s)$ whose forms are given in the Appendix. The amplitude of the relative velocity is given by

$$
\boldsymbol{w}_{i j}^{(0)}=\frac{(1-\lambda \nu)}{6 \pi \eta a_{j}} \boldsymbol{F}_{j}^{*}=-\frac{(1-\lambda \nu)}{6 \pi \eta a_{j}} k T \nabla \ln n_{j} .
$$

Now the relative diffusivity can be written in a similar form:

$$
\boldsymbol{D}_{i j}=D_{i j}^{(0)}[G(s) \hat{\boldsymbol{s}} \hat{\boldsymbol{s}}+H(s)(\boldsymbol{I}-\hat{\boldsymbol{s}} \hat{\boldsymbol{s}})],
$$


with

$$
D_{i j}^{(0)}=\frac{k T}{6 \pi \eta}\left(\frac{1}{a_{i}}+\frac{1}{a_{j}}\right) .
$$

The hydrodynamic functions $G(s)$ and $H(s)$ can be found in the Appendix.

The relative magnitude of the forcing by the chemical potential gradient compared with diffusion, which tries to maintain equilibrium, defines a Péclet number

$$
P e=\frac{\left|\boldsymbol{w}_{i j}^{(0)}\right|\left(a_{i}+a_{j}\right)}{2 D_{i j}^{(0)}}=\frac{(1-\lambda \nu)}{(1+\lambda)}\left|\hat{\nabla} \ln n_{j}\right| .
$$

In the linear-response regime that we consider here the Péclet number is small. From (4.51), we see that in addition to having a weak concentration gradient of solute, a new condition arises to ensure small $P e$, namely, $\lambda v \leqslant O(1)$, which, from (4.46) requires the solute to be so dilute that $\phi_{j} / \lambda^{2} \leqslant O(1)$. Now $\phi_{j} / \lambda^{2} \sim n_{j} a_{i}^{2} a_{j}$ and corresponds to the number of solute particles within one solute particle radius of the surface of particle $i$. The diluteness requirement is that there can only be one solute particle (on average) interacting with particle $i$.

The pair-distribution function can be written as

$$
p_{i j}=p_{i j}^{e q}\left(1+P e f_{i j}\right),
$$

and, to leading order in $P e, f_{i j}$ can be written in terms of a vector field

$$
f_{i j}=\boldsymbol{d}_{i j}(\boldsymbol{s}) \cdot \mathbf{1}_{\nabla n},
$$

where $\mathbf{1}_{\nabla n}$ is a unit vector in the direction of the solute gradient $\nabla n_{j}$. The steady Smoluchowski equation for $\boldsymbol{d}_{i j}$ becomes

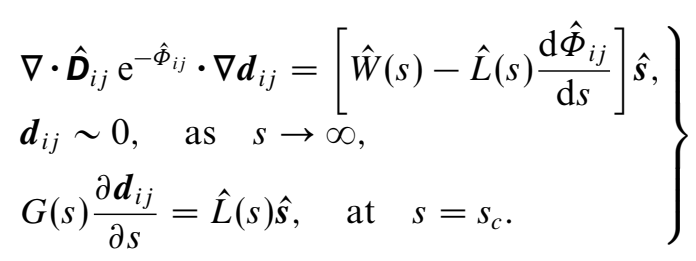

The hydrodynamic function $\hat{W}(s)=2(\hat{L}-\hat{M}) / s+\mathrm{d} \hat{L} / \mathrm{d} s$ comes from the divergence of the relative velocity. We have set the inner boundary condition at $s=s_{c} \geqslant 2$ rather than strictly at $s=2$ out of convenience when considering a hard-sphere potential as discussed below.

As a remark, we see now that departures from equilibrium are proportional to $P e$, which, from (4.51), is proportional to $\left|\nabla n_{j}\right|$. Using this perturbation in the back-flow expression (4.22) would generate terms proportional to $\left(\nabla n_{j}\right)^{2}$, which are nonlinear and violate the restriction to weak concentration gradients, i.e. linear response. Thus, it is necessary to use the equilibrium distribution in (4.22) as was done in arriving at the final expression (4.33).

The additional velocity of particle $i$ due to Brownian and interparticle forces in (4.43) is proportional to the departure of the pair-distribution function from equilibrium. Using (4.52), (4.43) becomes

$$
\left\langle\Delta \boldsymbol{U}_{i}\right\rangle=\frac{a_{i}^{2}}{\eta} k T\left|\nabla n_{j}\right|(1+\lambda)^{2}(1-\lambda v) \frac{1}{48 \pi} \int_{s \geqslant s_{c}}\left(\hat{\boldsymbol{M}}_{i i}-\hat{\boldsymbol{M}}_{i j}\right) \mathrm{e}^{-\hat{\phi}_{i j}} \cdot \nabla f_{i j} \mathrm{~d} \boldsymbol{s} .
$$


Integration by parts and introducing the $\boldsymbol{d}_{i j}$ field gives

$$
\begin{aligned}
&\left\langle\Delta \boldsymbol{U}_{i}\right\rangle=\frac{a_{i}^{2}}{\eta} k T \nabla n_{j}(1+\lambda)^{2}(1-\lambda \nu) \frac{1}{48 \pi} \cdot\left\{L\left(s_{c}\right) \mathrm{e}^{-\hat{\phi}_{i j}\left(s_{c}\right)} s_{c}^{2} \oint_{s_{c}} \boldsymbol{n} \boldsymbol{d}_{i j} \mathrm{~d} \Omega\right. \\
&\left.+\int_{s \geqslant s_{c}}\left(W(s)-L(s) \frac{\mathrm{d} \hat{\Phi}_{i j}}{\mathrm{~d} s}\right) \hat{\boldsymbol{s}} \boldsymbol{d}_{i j} \mathrm{~d} \boldsymbol{s}\right\},
\end{aligned}
$$

where $\nabla n_{j}$ is dotted into $\boldsymbol{d}_{i j}$. The two new hydrodynamic functions $L(s)$ and $W(s)$ are similar to $\hat{L}$ and $\hat{W}$, except they correspond to the motion of particle $i$ due to the forcing, rather than to the relative motion of particles $i$ and $j$; their forms can be found in the Appendix.

Equation (4.54) for the perturbation to the pair-distribution function and (4.56) for the additional velocity of particle $i$ apply for any size ratio, $\lambda$, and for any interparticle potential $\hat{\Phi}_{i j}$. We now discuss the two cases - with and without interparticle potential in $\$ \$ 4.5 .1$ and 4.5.2, respectively.

\subsubsection{No interparticle potential}

For no potential interactions between the $i$ particle and the solute we have $\hat{\Phi}_{i j}=0$ and the contact radius $s_{c}=2$. We refer to this situation as the 'pure hydrodynamic limit.' Owing to hydrodynamic lubrication interactions, the relative motion of two particles vanishes as the two particles come into contact. This implies $L(s) \sim 0$ as $s \rightarrow 2$ and the surface integral in (4.56) vanishes. The equations for $\boldsymbol{d}_{i j}$ and $\left\langle\Delta \boldsymbol{U}_{i}\right\rangle$ become

$$
\left.\begin{array}{l}
\nabla \cdot \hat{\boldsymbol{D}}_{i j} \cdot \nabla \boldsymbol{d}_{i j}=\hat{W}(s) \hat{\boldsymbol{s}}, \\
\boldsymbol{d}_{i j} \sim 0, \quad \text { as } \quad s \rightarrow \infty, \\
G(s) \frac{\partial \boldsymbol{d}_{i j}}{\partial s}=0, \quad \text { at } \quad s=2,
\end{array}\right\}
$$

and

$$
\left\langle\Delta \boldsymbol{U}_{i}\right\rangle=\frac{a_{i}^{2}}{\eta} k T \nabla n_{j}(1+\lambda)^{2}(1-\lambda v) \frac{1}{48 \pi} \cdot \int_{s \geqslant 2} W(s) \hat{\boldsymbol{s}} \boldsymbol{d}_{i j} \mathrm{~d} \boldsymbol{s} .
$$

Also note that $G(s) \sim(s-2)$ as $s \rightarrow 2$ and thus the boundary condition at contact becomes one of the limiting form of $\boldsymbol{d}_{i j}$.

The above still apply for any $\lambda$, but now we examine the small $\lambda$ limit in order to connect to the continuum analysis. The departure of the structure from equilibrium is driven exclusively by hydrodynamic interactions embodied in $\hat{W}(s)$, while the incremental velocity also depends on $W(s)$. From the known forms of the mobility functions for two particles,

$$
\hat{W}(s) \sim-\frac{120 \lambda}{1-\lambda \nu} \frac{1}{s^{5}}, \quad W(s) \sim \frac{120 \lambda^{3}}{s^{5}},
$$

and thus the incremental velocity scales as

$$
\left\langle\Delta \boldsymbol{U}_{i}\right\rangle=-\frac{a_{i}^{2}}{\eta} k T \nabla n_{j} \lambda^{4}[C+O(\lambda)]
$$

where the numerical constant $C$ needs to be determined by solving (4.57) and evaluating the integral of $W(s)$. Note that the integral is absolutely convergent as $\boldsymbol{d}_{i j} \sim 1 / s^{2}$.

The increment to the velocity of particle $i$ due to the contribution from Brownian motion vanishes as $\lambda^{4}$ for small $\lambda$ and is smaller than the back-flow contribution found in $\S 4.4$.1, which was $O\left(\lambda^{2}\right)$. Thus, as $\lambda \rightarrow 0$ the colloidal and continuum analyses agree 
that there is no motion of the $i$ particle due to a concentration gradient of solute if there are no potential interactions between the solute and the $i$ particle.

\subsubsection{Interparticle interaction: hard-sphere at $s=s_{c}=2(1+\Delta)$}

We now consider the case in which there are direct interparticle forces between the $i$ particle and the solute. If the interparticle potential is continuous everywhere, then (4.54) must be solved in the entire domain, and since the inner boundary condition is at hydrodynamic contact, $s=2, \hat{L}(2)=0$. This can require considerable work in general. However, if the interparticle potential is of the hard-sphere type at a distance $s_{c}=2(1+\Delta)$, as was used in $\S 4.4 .2$, then analytic progress is possible. And the most relevant case is when $\Delta \geqslant \lambda$, for now the separation between the surfaces of the phoretic and solute particles is not small compared with the size of the solute and the hydrodynamic functions take on simple forms. This is also the proper limit with which to compare the continuum analysis.

In the limit $\lambda \rightarrow 0$ for $\Delta \geqslant \lambda$ the hydrodynamic functions become $G, H \sim 1+O(\lambda)$ and $\hat{L} \sim 1+O(\lambda)$; the solute is much more mobile than the $i$ particle and so relative motion is just that for an isolated solute particle. Thus the perturbation to the pair-distribution function is governed by (with error of $O(\lambda)$ )

$$
\begin{aligned}
\nabla^{2} \boldsymbol{d}_{i j} & =0, \\
\boldsymbol{d}_{i j} & \sim 0, \quad \text { as } \quad s \rightarrow \infty, \\
\frac{\partial \boldsymbol{d}_{i j}}{\partial s} & =\hat{\boldsymbol{s}}, \quad \text { at } \quad s=s_{c},
\end{aligned}
$$

whose solution is a dipolar disturbance field

$$
\boldsymbol{d}_{i j}=-\frac{s_{c}^{3}}{2} \frac{s}{s^{3}} \text {. }
$$

Allowing for the change in notation, (4.64) gives the same concentration disturbance as found in the continuum description, (3.17). This is as it should be because the solute is so small that it freely moves in the solution surrounding the phoretic particle. Only if the solute can get close enough to the $i$ particle so that the gap spacing is smaller than the solute size, $r-\left(a_{i}+a_{j}\right)<a_{j}$, i.e. $\Delta<\lambda$, will it experience a reduction in its hydrodynamic mobility.

To evaluate the contribution to the velocity of particle $i$ we need $L$, which for small $\lambda$ is $L(s)=1-3 / s+4 / s^{3}$. This relative mobility function has the following interpretation: particle $i$ moves freely with unit mobility (the 1) due to the force acting on it. The equal and opposite force on particle $j$ generates a velocity field, $-3 / s+4 / s^{3}$, that entrains the $i$ particle in the opposite direction. Alternatively, one can interpret $L(s)$ as the normal component of the Stokes velocity field outside the spherical particle $i$. At the distance of closest approach $s_{c}=2(1+\Delta), L$ becomes

$$
L\left(s_{c}\right)=L(2(1+\Delta))=\frac{3}{2} \frac{\Delta^{2}\left(1+\frac{2}{3} \Delta\right)}{(1+\Delta)^{3}},
$$

which, for small $\Delta$, is just the Taylor series expansion of the velocity about the no-slip surface of particle $i$.

Since $W(s) \sim \lambda^{3}$, only the surface integral in (4.56) at $s=s_{c}$ contributes and the increment to the velocity of particle $i$ becomes

$$
\left\langle\Delta \boldsymbol{U}_{i}\right\rangle=-\frac{1}{6} \frac{a_{i}^{2}}{\eta} k T \nabla n_{j} \Delta^{2}\left(1+\frac{2}{3} \Delta\right)[1+O(\lambda)] .
$$


Combining this with the result from the back-flow contribution (4.41) in $\S 4.4 .2$, the velocity of the $i$ particle due to a concentration gradient of solute is given by

$$
\left\langle\boldsymbol{U}_{i}\right\rangle-\langle\boldsymbol{u}\rangle=-\frac{1}{2} \frac{a_{i}^{2}}{\eta} k T \nabla n_{j} \Delta^{2}\left(1+\frac{2}{3} \Delta\right)[1+O(\lambda)] .
$$

Restricting to short-range hard-sphere repulsion such that $\Delta \ll 1$ and making the identification of $a_{i} \Delta=b$, we recover precisely the continuum description phoretic velocity (3.35). And now we see clearly that the continuum result has contributions that arise both from the back-flow, 1/3, and from the indirect effect of Brownian and interparticle forces, $1 / 6$. And only in the latter did the disturbance to the solute concentration field caused by the impenetrability of the $i$ particle enter.

To summarize, we bring all the contributions together in one place for the two different cases considered. We have kept the indirect contribution from Brownian and interparticle forces separate from the back-flow contributions - those associated with the steady chemical potential gradients and the associated long-range hydrodynamic interactions discussed in $\S 4.1$ - as this distinction will become important in the next section.

(i) Case I: pure hydrodynamics, $\hat{\Phi}_{i j}=0, \lambda \rightarrow 0$ :

$$
\begin{aligned}
\left\langle\boldsymbol{U}_{i}\right\rangle-\langle\boldsymbol{u}\rangle & =-\frac{2}{9} \frac{a_{i}^{2}}{\eta} k T \nabla n_{j}\left[2 \lambda^{2}-\frac{5}{2} \phi_{j}+O\left(\lambda^{3}, \phi_{j} \lambda\right)\right], \\
\left\langle\Delta \boldsymbol{U}_{i}\right\rangle & =-\frac{a_{i}^{2}}{\eta} k T \nabla n_{j} \lambda^{4}[C+O(\lambda)] .
\end{aligned}
$$

(ii) Case II: excluded volume at $s_{c}=2(1+\Delta), \Delta \geqslant \lambda, \phi_{j} / \lambda^{2} \leqslant 1, \lambda \rightarrow 0$ :

$$
\begin{aligned}
\left\langle\boldsymbol{U}_{i}\right\rangle-\langle\boldsymbol{u}\rangle & =-\frac{1}{3} \frac{a_{i}^{2}}{\eta} k T \nabla n_{j} \Delta^{2}\left(1+\frac{2}{3} \Delta\right)[1+O(\lambda)], \\
\left\langle\Delta \boldsymbol{U}_{i}\right\rangle & =-\frac{1}{6} \frac{a_{i}^{2}}{\eta} k T \nabla n_{j} \Delta^{2}\left(1+\frac{2}{3} \Delta\right)[1+O(\lambda)] .
\end{aligned}
$$

It is reassuring that the continuum and colloidal perspectives give identical results. And it is now clear to what the continuum limit corresponds: the size of the solute is much smaller than the $i$ particle $\lambda=a_{j} / a_{i} \rightarrow 0$, the concentration of solute is very dilute, $\phi_{j}<\lambda^{2}$, and, as far as hydrodynamics are concerned, the solute generates the velocity field of an isolated spherical particle in Stokes flow (no hydrodynamic interactions between the solute and the $i$ particle, no lubrication) both for the backflow contribution of (4.22) and for the indirect contribution from Brownian and interparticle forces in (4.43); and it is essential to have both contributions.

But now it is also clear how to relax these restrictions. The colloidal perspective is valid for any size ratio $\lambda$, even if the solute is larger than the $i$ particle. In many situations of concentration gradient-induced motion, e.g. transport of proteins in biological applications, the solute may not be much smaller than the protein and the continuum perspective would not be applicable, and certainly there would be no 'thin interfacial layer'. The colloidal perspective can be used for any form of interparticle potential and can also be applied to the depletion flocculation of colloidal particles (Asakura \& Oosawa 1954). The colloidal perspective shows how hydrodynamics enter the picture and provides a rational starting point for including such interactions. It also provides a basis to go beyond a dilute solution and incorporate solute-solute interactions, perhaps via a Stokesian dynamics approach (Brady \& Bossis 1988). 
Attempting to incorporate these effects starting from the continuum perspective, for example, by using the Einstein viscosity in place of the solvent viscosity, is fraught with questions of consistency - if something is included in one place, must it be included in another place? (Please see $\S 6$ for a discussion of how to include solute-solute interactions.) And finally, there is now a rational basis for selecting the potential of interaction between the solute and phoretic particle that can in principle be measured experimentally, as is done in colloidal systems.

We close this section with some remarks and observations about Case II. Although restricted to small $\lambda$, the results are not limited to small $\Delta$. Indeed, they apply even for $\Delta \rightarrow \infty$, which gives a phoretic velocity that scales as $a_{i}^{2} \Delta^{3}$ ! How are we to understand this surprising behaviour? As discussed in $\S 4.1$ (see (4.8)), the chemical potential gradient driving force for the motion is proportional to the volume excluded to the centre of the solute. Thus, the appropriate length scale is the thermodynamic radius of the $i$ particle, $a_{t h}=a_{i}(1+\Delta)$, where what has been called up to now the radius, $a_{i}$, is the hydrodynamic radius - where the no-slip hydrodynamic boundary condition is applied. The driving force thus scales as $a_{t h}^{3}=a_{i}^{3}(1+\Delta)^{3}$. The mobility of the $i$ particle is set by hydrodynamics and scales inversely with the hydrodynamic radius. Thus, for large $\Delta$ the velocity of the $i$ particle scales as $a_{t h}^{3} / a_{i} \sim a_{i}^{2} \Delta^{3}$, as seen in Case II. This scaling applies to both the indirect contribution from Brownian and interparticle forces and the direct contribution from the back-flow. For the back-flow contribution there is no longer a cancellation of the large thermodynamic driving force and the fluid flow induced by the constant force on the solute: the solute is kept so far from the $i$ particle that the back-flow is weakened because of the $1 / r$ nature of the long-ranged hydrodynamic interactions. (The continuum approach will also produce this behaviour if the solute force density is confined to a thin layer at a distance of $O(a)$ from the particle surface, as now the curvature and finite size of the particle will be seen for the flow generated by this force density.) While a very large $\Delta$ may not be realistic, for a particle of $50 \mathrm{~nm}$ radius, interparticle interactions due to electrostatics (large double layers) or steric stabilization can easily result in a $\Delta \sim O(1)$, giving a phoretic velocity of $O\left(\left(a_{i}^{2} / \eta\right) k T \nabla n_{j}\right)$.

\section{Autonomous motion}

An important application of solute-induced particle motion is the autonomous motion of catalytic nanomotors (Paxton et al. 2004, 2006; Golestanian, Liverpool \& Ajdari 2005, 2007; Hong et al. 2007; Abecassis et al. 2008; Córdova-Figueroa \& Brady 2008; Ibele et al. 2009; Kagan et al. 2009; Wang et al. 2009; Ebbens \& Howse 2010). Here, a particle induces a gradient in a solute species by carrying out a chemical reaction at its surface. If the reaction is not uniform over the particle surface, then a net motion of the particle can result. This problem appears to have much in common with the phoretic motion of a particle due to a concentration gradient of solute, but, as shown below, there are important differences. The most notable is that the back-flow contribution is completely absent; only the indirect contribution from Brownian and interparticle forces remains. This raises questions about the continuum approach to autonomous motion employed by some authors (Golestanian et al. 2005, 2007).

To see why diffusiophoresis due to an external concentration gradient does not apply to autonomous motion, it is important to realize that the back-flow contribution arises because there is a constant statistical force $F_{j}^{*}=-\left(1 /\left(1-\phi_{j}\right)\right) \nabla \mu_{j}$ applied to each and every solute particle (and the solvent is force-free). In autonomous motion, however, 
there is no global macroscopic gradient of solute and thus no back-flow. To see this consider the following.

A reactive particle (particle $i$ ), which shall sometimes be called the 'motor', produces a concentration distribution of solute of the form

$$
n_{j}=n_{j}^{\infty}+\frac{s}{r}+\frac{\boldsymbol{p} \cdot \boldsymbol{x}}{r^{3}}+\cdots,
$$

where $n_{j}^{\infty}$ is the constant solute concentration far from the reactive particle, $s$ is the source (or sink) strength of the chemical reaction occurring on the particle surface, $\boldsymbol{p}$ is the dipole moment of the chemical reaction, etc.

The chemical potential gradient is

$$
-\nabla \mu_{j}=-k T \nabla \ln n_{j}=+k T \frac{s}{n_{j}^{\infty}} \frac{x}{r^{3}}+O\left(\frac{1}{r^{3}}\right),
$$

and thus the 'external' force applied to each solute particle decays as $1 / r^{2}$. The fluid velocity at the origin (the motor) due to this distribution of forces behaves as

$$
\boldsymbol{u}=\int \boldsymbol{J} \cdot\left(-\nabla \mu_{j}\right) n_{j} \mathrm{~d} V \sim k T s \int \boldsymbol{J} \cdot \frac{\boldsymbol{x}}{r^{3}} \mathrm{~d} V=0,
$$

where $\boldsymbol{J}$ is the Stokeslet. The integral is conditionally convergent (the integrand goes as $1 / r^{3}$ ) and by symmetry (or by detailed considerations as in $\S 4.2$ ) is zero.

The motion of the solute towards (or away from) the reactive particle creates a radial pressure gradient that drives fluid in the opposite direction to conserve volume. This spherically symmetric flow produces no net velocity of the reactive particle as illustrated in figure 2. The next term in the concentration field gives rise to an absolutely convergent integral $\left(\nabla \mu_{j} \sim 1 / r^{3}\right)$. This means that there is no back-flow integral and interactions between the solute and the motor are absolutely convergent and can be added in a pair-wise fashion.

From a continuum perspective, no macroscopic pressure gradient is needed to balance the 'weight' $\left(\boldsymbol{F}_{j}^{*}\right)$ of the solute. A similar situation arises in the sedimentation of a cloud of particles. A cloud of size $R$ composed of particles of size $a$ with a uniform number density $n$ falls (in Stokes flow) with velocity

$$
U^{\text {cloud }} \sim \frac{\left(\Delta \rho \frac{4}{3} \pi a^{3} g\right) n \frac{4}{3} \pi R^{3}}{6 \pi \eta R} \sim \frac{2}{9} \frac{\Delta \rho g}{\eta} \phi R^{2},
$$

showing that the velocity grows as the size, $R$, squared. The larger the cloud, the faster it falls. The fluid displaced by the cloud flows back up around, not through, the cloud, as illustrated in figure 3. There is no black-flow hindrance to the motion; there is no average pressure gradient. In contrast, in a suspension of heavy particles there is no place to flow 'around' the particles. There is a uniform pressure gradient that balances the weight of the particles and this hinders the motion. A suspension falls slower than an isolated particle (see (4.23)), while a cloud falls faster. In the context of the chemical potential gradient force, the important feature of the gradient is not that it extends to infinity, for surely one would encounter the container boundaries at some distance, but rather its one-dimensional nature - there is no place to 'flow around': the back-flow of fluid must flow uniformly up against the driving force.

For autonomous particle motion, since there is no back-flow contribution only the indirect contribution arising from Brownian and interparticle forces, (4.43), can give rise to net motion. The pair-distribution satisfies the same Smoluchowski equation as 


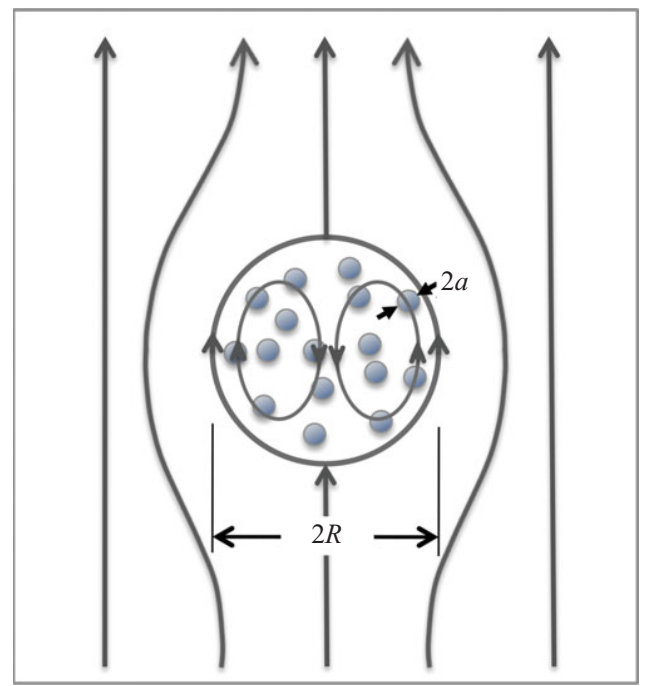

FIgURE 3. (Colour online) Sketch of a cloud of size $R$ composed of heavy particles of size $a$ falling due to gravity. There is no uniform back-flow of fluid as the fluid displaced by the falling cloud flows back up around, not through, the cloud. The particles within the cloud recirculate just like the interior fluid of a viscous drop (Nitsche \& Batchelor 1997).

before, (4.44), except the boundary condition at contact now becomes one of the flux of particle $j$ relative to the reactive particle $i$ being given by the rate of production of $j$ due to chemical reaction, $r_{j}$.

Scaling as before and separating out the equilibrium distribution, (4.44) becomes

$$
\left.\begin{array}{l}
\nabla \cdot \hat{\boldsymbol{D}}_{i j} \mathrm{e}^{-\hat{\Phi}_{i j}} \cdot \nabla f_{i j}=P e \nabla \cdot \hat{\boldsymbol{w}}_{i j} \mathrm{e}^{-\hat{\Phi}_{i j}}\left(1+f_{i j}\right), \\
f_{i j} \sim 0, \quad \text { as } \quad s \rightarrow \infty, \\
G(s) \frac{\partial f_{i j}}{\partial s}-P e \boldsymbol{n} \cdot \hat{\boldsymbol{w}}_{i j}\left(1+f_{i j}\right)=\frac{\left(a_{i}+a_{j}\right)}{2 D_{i j}^{(0)}} \mathrm{e}^{\hat{\Phi}_{i j}} r_{j}, \quad \text { at } s=s_{c} .
\end{array}\right\}
$$

The relative velocity in (4.44) is now $\boldsymbol{w}_{i j}=-\left\langle\boldsymbol{U}_{i}\right\rangle \cdot \hat{\boldsymbol{w}}_{i j}=-\left\langle\boldsymbol{U}_{i}\right\rangle \cdot\left(\hat{\boldsymbol{M}}_{i i}-\hat{\boldsymbol{M}}_{i j}\right)$, where $\left\langle\boldsymbol{U}_{i}\right\rangle$ is the sought-after particle velocity and is not given a priori but must be found as a result of the calculations. In (5.5) the Péclet number is

$$
P e=\frac{U_{i}\left(a_{i}+a_{j}\right)}{2 D_{i j}^{(0)}}=\frac{U_{i} a_{i}}{2 D_{j}^{(0)}},
$$

where $D_{j}^{(0)}=k T / 6 \pi \eta a_{j}$ and $U_{i}$ is the magnitude of the sought-after velocity of particle $i$.

For the reaction we take as a simple example a first-order, irreversible reaction

$$
r_{j}=-\kappa p_{i j} h(\hat{\boldsymbol{s}}),
$$

where $h(\hat{\boldsymbol{s}})$ sets the structure of the non-uniform reaction on the particle surface and $\kappa$ is the reaction velocity. The ratio of the reaction velocity to diffusion defines the Damköhler number

$$
D a=\frac{\kappa a_{i}}{2 D_{j}^{(0)}}
$$


It is important to note that if we have pure hydrodynamics, i.e. no interparticle forces, then since the relative flux of the $i-j$ pair vanishes at hydrodynamic contact $r=a_{i}+a_{j}$, there can be no effect of the surface chemical reaction. A surface chemical reaction must take place at some offset distance $r=r_{c}>a_{i}+a_{j}$ so that a finite flux can supply reactant to the particle surface.

Now we take the small $\lambda$ limit and have the reaction occur at the hard-sphere distance $s_{c}=2(1+\Delta)$ as before. Defining a new independent variable $\tilde{s}=s / s_{c}$ to transform the contact condition to $\tilde{s}=1$, the concentration distribution satisfies

$$
\left.\begin{array}{l}
\nabla^{2} f_{i j}=-\tilde{P} e\left\langle\hat{\boldsymbol{U}}_{i}\right\rangle \cdot \hat{\boldsymbol{w}}_{i j} \cdot \nabla f_{i j}, \\
f_{i j} \sim 0, \quad \text { as } \tilde{\boldsymbol{s}} \rightarrow \infty, \\
\frac{\partial f_{i j}}{\partial s}=-\tilde{D a}\left(1+f_{i j}\right) h(\hat{\boldsymbol{s}})-\tilde{P} e \boldsymbol{n} \cdot\left\langle\hat{\boldsymbol{U}}_{i}\right\rangle L(\Delta)\left(1+f_{i j}\right), \quad \text { at } \quad \tilde{\boldsymbol{s}}=1,
\end{array}\right\}
$$

where the Péclet and Damköhler numbers are now based on the thermodynamic radius

$$
\tilde{P} e=\frac{U_{i} a_{i}(1+\Delta)}{D_{j}^{(0)}} \quad \text { and } \quad \tilde{D a}=\frac{\kappa a_{i}(1+\Delta)}{D_{j}^{(0)}},
$$

$\left\langle\hat{\boldsymbol{U}}_{i}\right\rangle$ is a unit vector in the direction of the autonomous motion that is determined by the asymmetric distribution of reaction on the motor surface embodied in function $h(\hat{\boldsymbol{s}})$, and the hydrodynamic function $L(\Delta)$ is given by $(4.65)$. The average velocity of the reactive particle from (4.43) becomes

$$
\left\langle\boldsymbol{U}_{i}\right\rangle=\frac{n_{j}^{\infty} k T\left[a_{i}(1+\Delta)\right]^{2}}{6 \pi \eta a_{i}} L(\Delta) \oint_{\tilde{s}=1} \boldsymbol{n} f_{i j}(1 ; \tilde{D a}, \tilde{P} e, L(\Delta)) \mathrm{d} \Omega .
$$

Equation (5.11) for the velocity of an autonomous reactive particle has a suggestive form: the 'solute pressure', $n_{j}^{\infty} k T$, acts on the thermodynamic area of the particle $\left[a_{i}(1+\Delta)\right]^{2}$ to generate a force. The net strength of this force is determined by the distribution of solute over the surface of the particle - the integral of $f_{i j}$ - and modulated in amplitude by the hydrodynamic function $L(\Delta)$. This net solute force is balanced by the Stokes drag on the particle, $6 \pi \eta a_{i}\left\langle\boldsymbol{U}_{i}\right\rangle$, to give the resultant velocity. From a microscopic picture, each solute particle gives a thermal kick $(k T)$ upon collision with the motor. The hydrodynamic function modulates the kick amplitude displacing the fluid between the solute and the motor reduces the impulse. If there are more kicks on one side of the motor than the other there will be net motion of the motor, with Stokes drag balancing the net solute force.

Equations (5.9)-(5.11) are the same as those used by Córdova-Figueroa \& Brady (2008) in their treatment of self propulsion due to surface chemical reactions. CórdovaFigueroa and Brady neglected hydrodynamic interactions by taking $\Delta \geqslant O(1)$ so that $L(\Delta) \approx 1$ and $\hat{\boldsymbol{w}}_{i j} \approx \boldsymbol{I}$. They did indicate where (4.43) could be found, however (Russel et al. 1989; Squires \& Brady 2005; Khair \& Brady 2006), which is all that is necessary for including hydrodynamics. Thus, the criticisms levelled at their treatment, which are based on the mistaken assumption that a back-flow-like contribution plays a role in autonomous motion (Jülicher \& Prost 2009a,b), are misplaced. Indeed, CórdovaFigueroa and Brady went further and explicitly considered the importance of both reactants $(R)$ and products $(P)$ on the net force, showing that for the reaction, $R \rightarrow \theta P$, where $\theta$ is the stoichiometry of the reaction, (5.11) must be multiplied by the factor $\left(1-\theta D_{R} / D_{P}\right)$. This stoichiometry/diffusivity factor tells how many products are 
produced per reactant, $\theta$, and how fast the reactants diffuse relative to the products, $D_{R} / D_{P}$, and thus gives both the number and amplitude of the thermal kicks given by the reactants/products to the motor. As discussed below, to include hydrodynamics one only needs to take the results of Córdova-Figueroa \& Brady and multiply by the factor $L(\Delta)$.

There should be a way to rescue the continuum perspective for treating reactiondriven autonomous motion, but simply supposing that the phoretic velocity formula, (3.34), which is based on the presumption of a 'imposed' global concentration gradient and associated back-flow pressure gradient, can be used without derivation (Golestanian et al. 2005, 2007) is not justified. The change needed in the continuum treatment is that there is no imposed concentration gradient: rather, a chemical reaction occurs at the reactive particle surface and creates a concentration gradient of reactants (and products). In the thin interfacial limit, this means that the solute distribution outside the interfacial layer now has a flux condition $\boldsymbol{n} \cdot \boldsymbol{j}^{\text {out }}=r_{j}$, but the reaction rate depends on the solute concentration at the surface within the interfacial layer. The solution for the solute distribution within the layer will then give the pressure distribution from (3.26) and then the slip velocity from (3.29). Note that this presupposes that there is an interactive force, $\boldsymbol{f}_{s}$, between the motor and the solute (the reactant). Just having a gradient in solute chemical potential does not generate any solution motion, and therefore no motion of the motor, because the Gibbs-Duhem relation (4.1) shows that the net chemical potential gradient force on a volume element is zero. (In the colloidal treatment this manifests itself as the vanishing of the flux at hydrodynamic contact, as was discussed leading up to (5.9).) While one could thus 'rescue' the continuum treatment, such a rescue is not needed; (5.9)-(5.11) are all that is necessary.

Before closing this section we discuss the applicability of this reaction-diffusion model for chemical-reaction-induced motion. It is certain that the colloidal description breaks down at the motor surface, where the actual chemical reaction of the molecular species takes place. In a gas the breaking of a chemical bond would be accompanied by the recoil of the reactants and products. In a condensed liquid, however, upon reaction any energy released (or consumed in an endothermic reaction) would be immediately transferred to (from) the solvent in the form of heat. A product species would travel at most a few solvent molecular sizes before thermalizing with the solvent. While this could lead to local heating (or cooling) of the solvent, we have neglected this effect presuming that the conduction of heat by the solvent is sufficiently rapid, i.e. large Lewis numbers $L e=\alpha / D \gg 1$, which is typical of liquids; here, $\alpha$ is the thermal diffusivity of the solvent. Further, a particle that receives a thermal kick at low Reynolds number travels a small fraction of its size (proportional to the Reynolds number) and thus direct momentum exchange between the reactants/products and the motor does not lead to any significant motion. Over time the chemical reaction establishes variations in the concentration of reactants and products on the scale of the motor which can lead to the diffusiophoretic motion of the motor. Hence, a colloidal-scale modelling of the process may be applicable. Diffusiophoresis is an entropy-driven motion and thus the detailed nature of the chemical reaction (other than the stoichiometry/diffusivity factor $\left.\left(1-\theta D_{R} / D_{P}\right)\right)$ is not important.

In closing this section we summarize here for convenience the main results of Córdova-Figueroa \& Brady (2008), who considered a particle with reaction on half of its surface, and extend these results to include the effects of hydrodynamic interactions. For small Péclet numbers the solute or reactant just diffuses in the solution, and the distribution of solute, $f_{i j}$ in (5.9) and (5.11), depends only on the Damköhler number. 
For small $\tilde{D a}=\kappa a_{i}(1+\Delta) / D_{j}^{(0)} \ll 1, f_{i j}$ is proportional to $\tilde{D a}$ and (5.11) gives

$$
\left.\begin{array}{rl}
\left|\left\langle\boldsymbol{U}_{i}\right\rangle\right| & \sim \frac{n_{j}^{\infty} k T\left[a_{i}(1+\Delta)\right]^{2}}{6 \pi \eta a_{i}} L(\Delta) \tilde{D a}, \\
& \sim n_{j}^{\infty} a_{i}^{2} a_{j} \Delta^{2} \frac{3}{2}\left(1+\frac{2}{3} \Delta\right) \kappa, \\
& \sim \phi_{j}^{\infty} \frac{3}{2}\left(\frac{\Delta}{\lambda}\right)^{2}\left(1+\frac{2}{3} \Delta\right) \kappa,
\end{array}\right\}
$$

where we have now included the hydrodynamic factor, $L(\Delta)$. Thus, for small $\tilde{D a}$ the speed is proportional to the volume fraction of solute and to the reaction velocity $\kappa$, but is independent of the fluid viscosity, the thermal energy and the size of the motor. Typical values will depend, of course, on the concentration of reactant and the nature of the chemical reaction, e.g. the reaction need not be first-order and irreversible as in (5.7). (The motor velocity is also proportional to the stoichiometry/diffusivity factor $\left(1-\theta D_{R} / D_{P}\right)$.)

At the other extreme of high Damköhler number the reaction at the motor surface is diffusion-limited and the concentration on the reactive portion of the surface falls to zero, while remaining approximately $n_{j}^{\infty}$ on the non-reactive half (see CórdovaFigueroa \& Brady 2008). Thus, (5.11) gives

$$
\left.\begin{array}{rl}
\left|\left\langle\boldsymbol{U}_{i}\right\rangle\right| & \sim \frac{n_{j}^{\infty} k T\left[a_{i}(1+\Delta)\right]^{2}}{6 \pi \eta a_{i}} L(\Delta) \\
& \sim \frac{n_{j}^{\infty} k T a_{i}}{6 \pi \eta} \frac{3}{2} \Delta^{2} \frac{1+(2 / 3) \Delta}{1+\Delta} \\
& \sim \phi_{j}^{\infty} \frac{3}{2}\left(\frac{\Delta}{\lambda}\right)^{2} \frac{1+(2 / 3) \Delta}{1+\Delta} \frac{D_{j}^{(0)}}{a_{i}} .
\end{array}\right\}
$$

At high Damköhler number the speed is proportional to the solute pressure, $n_{j}^{\infty} k T$, the motor size, $a_{i}$, and inversely proportional to the solvent viscosity, $\eta$, but independent of the reaction rate $\kappa$ (and of its specific form because $f_{i j}$ will be zero at the surface whenever diffusion-limited). Or, the speed can be written in terms of the volume fraction of solute and the diffusive velocity of the solute $D_{j}^{(0)} / a_{i}$.

For all $\tilde{D a}$ at small $\tilde{P e}$ the motor velocity is proportional to the concentration of solute (or reactant), $\phi_{j}^{\infty}$, and to the ratio of the non-dimensional interactive scale $\Delta$, which is set by the physico-chemical interactions between the motor and the solute, to the size of the solute, $(\Delta / \lambda)^{2}$. While in many catalytic nanomotor situations both $\Delta$ and $\lambda$ may be small, their ratio, which is the range of the solute-motor interactive length compared to the solute size, is not; $\Delta / \lambda$ can be $O(10)$ or larger.

The above results are valid for small Péclet numbers, where the advective motion of the motor does not influence the solute concentration profile. In the experiments of Paxton et al. (2004) and Howse et al. (2007), the decomposition of hydrogen peroxide on the platinum half of a micrometre-sized motor resulted in speeds of a few microns per second for reactant concentrations of a few per cent. This gives a Péclet number $\tilde{P} e=U_{i} a_{i}[1+\Delta] / D_{j}^{(0)}=U_{i} a_{i}[1+\Delta] / D_{i}^{(0)} \times \lambda \approx 10 \lambda$, since the Stokes-EinsteinSutherland diffusivity of a micrometre-sized particle is approximately $1 \mu \mathrm{m}^{2} \mathrm{~s}^{-1}$. Thus, while not necessarily very large, Péclet numbers of $O(1)$ could be achieved in practice. Further, both estimates at small and large $\tilde{D a},(5.12)$ and (5.13), predict the speed to increase linearly with the concentration of solute and thus the Péclet number can, in 
principle, become large and it may no longer be appropriate to neglect the effects of convection on the distribution of the solute.

Córdova-Figueroa \& Brady (2008) considered the effects of finite Péclet number on the distribution of solute and thus on the motor speed. The problem must now be solved self-consistently, as one does not know the motor speed a priori but it must be found from (5.11), which depends on $f_{i j}$, which in turn depends on $\tilde{P} e$ in (5.9). Equation (5.11) gives a self-consistent expression for the unknown motor speed or Péclet number

$$
\tilde{P e}=\Gamma\left\langle\hat{\boldsymbol{U}}_{i}\right\rangle \cdot \oint_{\tilde{s}=1} \boldsymbol{n} f_{i j}(1 ; \tilde{D a}, \tilde{P} e, L(\Delta)) \mathrm{d} \Omega
$$

where

$$
\Gamma=\phi_{j}^{\infty} \frac{3}{2}\left(\frac{\Delta}{\lambda}\right)^{2}\left(1+\frac{2}{3} \Delta\right),
$$

is a non-dimensional measure of the concentration of the solute. For a given reaction stoichiometry and solute-motor interactive scale $\Delta$, the parameters that can be varied are the Damköhler number, $\tilde{D} a$, and the 'concentration' of the solute as expressed by $\Gamma$. The speed of the motor or Péclet number, $\tilde{P} e$, is then an output. Note that $\Gamma$ is precisely the concentration factor appearing in the expressions for the motor speed at low $\tilde{P} e,(5.12)$ and (5.13).

Córdova-Figueroa \& Brady (2008) examined the behaviour for all $\Gamma$ neglecting hydrodynamic interactions, i.e. for $L(\Delta)=1$, and showed that rather than the speed increasing with $\Gamma$ (or with concentration), a maximum Péclet number is obtained whose value depends on $\tilde{D} a$. The maximum $\tilde{P} e$ is not very high, typically being of $O(10)$. The reason the motor speed is limited is the following: the motor moves in the direction of lower solute concentration, being propelled along by collisions with solute particles on the non-reactive portion of its surface. If the motor were to move too fast - faster than the solute can diffuse - it would leave behind the solute that was propelling it in the first place. Thus, the motor speed is limited by the diffusive velocity of the solute $D_{j}^{(0)} / a_{i}$. This self-regulation also has the consequence that the motor speed becomes independent of the solute concentration at high $\Gamma$. Suppose the motor speed were to increase as the concentration increased, then the motor would leave behind the solute that drives it and thus must slow down so that there is a precise balance. (The opposite would be the case if the motor were to slow down upon increasing the concentration: the increased concentration on the nonreactive portion would lead to more collisions which would increase the speed.) This self-regulating mechanism will also be operative with hydrodynamic interactions, although the detailed values of the maximum $\tilde{P} e$ will depend on $L(\Delta)$. Thus, we predict the following:

$$
\left|\left\langle\boldsymbol{U}_{i}\right\rangle\right| \sim\left\{\begin{array}{ll}
\kappa, & \tilde{D} a \rightarrow 0, \\
D_{j}^{(0)} / a_{i}, & \tilde{D a} \rightarrow \infty,
\end{array} \text { for } \quad \Gamma \gg 1 .\right.
$$

The motor speed becomes independent of the solute concentration at high $\Gamma$, whereas it is directly proportional to the concentration at low $\Gamma$, and varies monotonically from one limit to the other. The value of $\Gamma$ at which this limiting speed is reached depends on $\tilde{D a}$, being as low as $\Gamma \approx 10$ for $\tilde{D a}=0.1$ to $\Gamma \approx 500$ for $\tilde{D a}=100$ (Córdova-Figueroa 2008). 
Córdova-Figueroa \& Brady (2008) obtained the same results (5.12)-(5.16) neglecting hydrodynamic interactions between the motor and the solute. Apparently, the only effect of hydrodynamics is to introduce the factor $\Delta^{2}$ in the definition of $\Gamma$.

\section{Conclusions}

We have provided both continuum and colloidal perspectives on the motion of particles driven by solute gradients and shown that the two perspectives precisely agree in their common domain of applicability, which is when the solute size is much smaller than that of the phoretic particle, $\lambda \rightarrow 0$, and when the solute concentration is very dilute, $\phi_{s} \rightarrow 0$. While the two approaches agree, they view the problem from two different levels of description. The continuum perspective uses non-equilibrium thermodynamics and continuum hydrodynamics and has the requirement for phoretic motion that the total force on the particle is zero. This force is a sum of the continuum hydrodynamic stress and an interactive force with the solute. At the colloidal level one is down amongst the solute particles which are undergoing Brownian motion. The dynamics at this scale are governed by the equations of colloidal dynamics, with the Smoluchowski equation determining the distribution of the solute about the phoretic particle. At this scale, there are two distinct contributions to the motion of the phoretic particle: (i) a 'back-flow' contribution composed of the motion of the particle due to the chemical potential gradient force acting on it and a compensating fluid motion driven by the long-range hydrodynamic velocity disturbance caused by the chemical potential gradient force acting on all the solute particles, and (ii) an indirect contribution arising from the mutual interactive and Brownian forces on the solute and the phoretic particle, that contribution being non-zero because the distribution of solute about the phoretic particle has been driven out of equilibrium by the chemical potential gradient of the solute. At the colloidal level the forces acting on the phoretic particle - both the statistical or 'thermodynamic' chemical potential gradient and Brownian forces and the interparticle force - are balanced by the Stokes drag of the solvent to give the net phoretic velocity.

The fact that at the colloidal level there can be statistical or 'thermodynamic' forces acting on the particles seems to have caused confusion with some authors (Jülicher \& Prost 2009a,b), who approach phoretic motion from the macroscopic perspective where the statistical forces do not appear. But, as shown explicitly by detailed analysis, there is no disagreement. They are two different levels of description of the same phenomenon and both can be correct. In the continuum perspective one often discusses the form of the velocity disturbance caused by a phoretic particle. In Stokes flow a particle subject to a net force produces a velocity that decays as $1 / r$, and therefore force-free phoretic motion should have velocity fields that decay faster. Indeed, an autonomous or self-phoretic particle must have zero force and torque and therefore a velocity field that decays at least as fast as a symmetric force dipole or stresslet, which decays as $1 / r^{2}$. In the classic problem of phoresis (thermal, electrical, etc.) the velocity disturbance decays as $1 / r^{3}$, a potential dipole. However, this $1 / r^{3}$ behaviour is the result of the 'thin interfacial layer' condition (and only when $\boldsymbol{n} \cdot \nabla n^{\text {out }}=0$ ) and is not the behaviour in general. For 'thick' layers - e.g. thick double layers in electrophoresis - the velocity disturbance caused by the phoretic particle decays as $1 / r$ until one is outside the double-layer thickness, which can be arbitrarily far from the particle. The condition of phoretic motion is not one of zero hydrodynamic force, but rather one of zero total force. 
At the colloidal level one does not need to ask how the velocity field decays; the full Stokes-flow hydrodynamics are included rigorously from the outset. However, one can show that the velocity disturbance far from the phoretic particle has the same structure as in the continuum treatment. The back-flow contribution has all the particles 'settling' owing to the solute chemical potential gradient driving force. Although the large particle experiences a force and creates a velocity disturbance that decays as $1 / r$, this flow is cancelled by the back-flow velocity disturbance due to all the solute particles - that is the whole point of the proper summation of the long-range interactions. Since the $1 / r$ Stokeslet is cancelled, the next term is the force quadrupole (or potential dipole), $\nabla^{2} \boldsymbol{J}$ (see Faxen's law (4.17)), which decays as $1 / r^{3}$. (The torque and average symmetric force dipole or stresslet are zero for the spherical particles considered here. In general, one needs to extend the back-flow expressions to include these terms; these expressions can be found in Brady et al. 1988.) The indirect Brownian and interparticle forces contribution from (4.55) produces a velocity disturbance proportional to $\left(\hat{\boldsymbol{M}}_{i i}-\hat{\boldsymbol{M}}_{i j}\right) \cdot \nabla f_{i j} \sim 1 / r^{3}$, owing to the dipolar disturbance to the pair-distribution function (4.64). Thus, the colloidal perspective produces the same velocity disturbance decay as in the continuum treatment.

The colloidal perspective offers several advantages over the classical continuum approach, not least of which is to be able to relax the conditions that the solute must be small compared to the phoretic particle and it must be dilute. The equations we have presented in $\S 4$ are valid for any size ratio and for any concentration. This is important because when trying to incorporate non-dilute solute effects in the continuum treatment one is always left worrying about consistency. If the chemical potential in the flux expression (3.4) is modified to include solute-solute interactions does one also need to modify the diffusivity and the viscosity? And if so, how? These issues are discussed next.

The correct form for the continuum solute flux that includes solute-solute interactions is

$$
\boldsymbol{j}=n \boldsymbol{v}+n \boldsymbol{M}\left(\phi_{s}\right) \cdot\left(\boldsymbol{f}_{s}-\frac{1}{1-\phi_{s}} \nabla \mu\right),
$$

which is to be used in the solute conservation (3.3). In (6.1) $\boldsymbol{M}\left(\phi_{s}\right)$ is the collective mobility of the solute, giving the average 'sedimentation' velocity of a solute particle subject to an external force. For a dilute solute, $\phi_{s} \rightarrow 0, k T \boldsymbol{M}=D \boldsymbol{I}$ with $D$ the StokesEinstein-Sutherland diffusivity of a isolated solute particle.

The driving forces for the motion relative to the mean advective flux, $n \boldsymbol{v}$, are the external force (or the interactive force with a larger phoretic particle) $\boldsymbol{f}_{s}$ and the gradient of the solute's chemical potential

$$
\mu=k T \ln n+\mu_{e x c}\left(\phi_{t h}\right),
$$

where $\mu_{\text {exc }}\left(\phi_{t h}\right)$ is the 'excess' chemical potential that accounts for solute-solute thermodynamic interactions. Note that the chemical potential depends on the thermodynamic volume fraction of solute, $\phi_{t h}$, while the collective mobility depends on the actual or hydrodynamic volume fraction, $\phi_{s}$, of the solute; in general, $\phi_{t h} \geqslant \phi_{s}$. For example, solute particles may be kept apart by steric interactions such that their thermodynamic radius is greater than their actual (or hydrodynamic) radius, $a_{t h}>a_{s}$.

The continuum solution stress that must be used with this expression for the solute flux in Cauchy's equation of motion (3.5) is

$$
\boldsymbol{\Sigma}=-\left[\left(1-\phi_{s}\right)\left\langle p_{f}\right\rangle+\Pi\left(\phi_{t h}\right)\right] \boldsymbol{I}+2 \eta_{e f f}\left(\phi_{t h}\right) \boldsymbol{e},
$$


where the solute pressure is

$$
\Pi\left(\phi_{t h}\right)=n k T+\Pi_{e x c}\left(\phi_{t h}\right),
$$

with the 'excess' solute pressure depending on the thermodynamic volume fraction. The average pressure in the fluid, $\left(1-\phi_{s}\right)\left\langle p_{f}\right\rangle$, is determined by the incompressibility constraint on the solution velocity $\nabla \cdot v=0$, and thus one may simply write $p=(1-$ $\phi)\left\langle p_{f}\right\rangle+\Pi\left(\phi_{t h}\right)$ in the solution stress as this pressure, $p$, will be what is determined from Cauchy's equation of motion for the incompressible solution. At small solute Péclet number the deviatoric stress is of Newtonian form, with the effective viscosity being the steady zero-shear-rate viscosity,

$$
\eta_{\text {eff }}=\eta\left[1+\frac{5}{2} \phi_{s}+\eta_{r}\left(\phi_{t h}\right)\right],
$$

containing the Einstein viscosity correction and the 'excess' relative shear viscosity, which depends on the thermodynamic volume fraction.

Given the solute-solute interparticle interactions (e.g. hard-sphere, van der Waals, etc.) the thermodynamic quantities, $\mu$ and $\Pi$, are well-defined and known (or can easily be computed) for all thermodynamic volume fractions $\phi_{t h}$ (although $\Pi\left(\phi_{t h}\right)$ is not really needed because of incompressibility). The two transport coefficients, $\boldsymbol{M}\left(\phi_{s}\right)$ and $\eta_{e f f}\left(\phi_{t h}\right)$, are also well known either from theory, simulations or experiments for sedimentation and the linear viscoelastic behaviour, respectively (Brady \& Bossis 1988; van der Werff \& de Kruif 1989; Ladd 1990; Brady 1993; Foss \& Brady 2000). Note that (6.1)-(6.3) are the consistent set of constitutive relations to be used - that is, if $O\left(\phi_{t h}^{2}\right)$ terms are retained in the chemical potential, then they should also be retained in the shear viscosity.

While the generalized expressions above can be used for non-dilute solute concentrations, there still are issues with the continuum approach to phoretic motion. Even if the phoretic particle is much larger than the solute and so appears as a macroscopic object, boundary conditions are needed for the solute concentration and the solution velocity. No flux of solute through the phoretic particle may be valid, but no slip of the solution velocity is not, in general, valid. While the solvent or fluid satisfies no slip on solid surfaces, the volume averaged suspension or solution velocity, $\boldsymbol{v}=\langle\boldsymbol{u}\rangle$, does not. The proper boundary condition on the solution velocity needs to be derived and/or modelled. Further, the expressions for the solute flux and solution stress apply to a volume element containing many solute particles so that an average can be formed at a 'continuum point'. Implicit in the continuum treatment is that the spacing between solute particles is small compared with their distance to any boundaries so that interactions between solute particles dominate over those between a solute particle and the boundary - small Knudsen numbers. But in many situations of phoretic motion the interaction length of the solute-phoretic interactive potential will be of order the solute size or the solute-solute spacing and therefore the Knudsen number would not be small. For example, in the solute flux one needs $\langle\boldsymbol{M} \cdot \boldsymbol{F}\rangle$, which is not equal to $\langle\boldsymbol{M}\rangle \cdot\langle\boldsymbol{F}\rangle$ when interactions with a boundary are important. So just where non-dilute solute concentration effects would be of most interest - near the phoretic particle surface - the continuum treatment breaks down.

At the colloidal level there is no breakdown as specific interactions, both thermodynamic and hydrodynamic, between the solute particles and the boundary (the phoretic particle) are properly taken into account at the outset. Further, couplings between the thermodynamic and hydrodynamic effects are also naturally included. It may be difficult to make analytical progress beyond the dilute limit, but numerical Stokesian dynamics approaches are certainly possible. And note that with Stokesian 
dynamics, since one is computing directly the motion of all the Brownian particles (just as one does in molecular dynamics) there is no limitation of being near equilibrium or in the linear response regime. Indeed, there is no need to impose a chemical potential gradient of the solute; the Brownian motion of the solute particles will generate the proper statistics. The colloidal perspective also offers a rational starting point for considering fluctuations in behaviour - how does a phoretic particle undergo a random walk or diffuse in addition to its directed motion? It may also provide a starting point for going beyond the mean-field treatment of electrokinetics - manybody electrostatics are very similar to many-body hydrodynamics.

We also saw how the colloidal perspective is able to address autonomous motion or self-phoresis. And here the important point is that the back-flow contribution is absent because there is no imposed global chemical potential gradient; only the indirect Brownian and interparticle forces contribution, (4.43), is present. It was shown that the velocity of the particle resulting from the Brownian and interparticle forces contribution could be written in terms of a mobility times the integral of the local solute pressure over the surface of contact between the particle and the solute (see (2.7)), recovering precisely the result of Córdova-Figueroa \& Brady (2008) in their analysis of self-propulsion. Further, it was shown how one could include hydrodynamic interactions by scaling Córdova-Figueroa \& Brady's results with the hydrodynamic function $L(\Delta)$ as discussed in $\S 5$ (see (5.12)-(5.16)). The use of the word 'osmotic' by Córdova-Figueroa \& Brady (2008), which is a well-accepted and defined term for colloidal dispersions, for the 'solute' pressure may have caused some confusion to those accustomed to working at the continuum level, where the macroscopic global solute pressure gradient is balanced by the fluid pressure gradient. There is no inconsistency, as there is no imposed chemical potential gradient for autonomous motion and thus no back-flow or macroscopic solute pressure gradient. The asymmetric chemical reaction definitely creates a local variation in the solute concentration and thus in the solute pressure which is responsible for the motion.

Finally, the presence or lack of back-flow may have implications for other transport processes. For example, the motion of a particle confined to an interface and driven by surface tension gradients may be such a case. From a continuum perspective, a gradient in the concentration of a surfactant molecule gives rise to a surface tension gradient which in turn gives rise to a Marangoni stress that drives fluid motion within and parallel to the interface. A particle in the interface will then be entrained in this Marangoni flow. From a 'colloidal' perspective at the level of the surfactant molecules (particles), the back-flow of fluid induced by the chemical potential gradient of the surfactant need not flow back through the interface itself, but rather the fluid would return through the layers of fluid on either side of the interface. Thus, a nonsurfactant particle in the interface (or a surfactant particle for that matter) would experience an entropic force proportional to its area, as this is the space excluded to the surfactant (the solute). But now the motion of this particle will not be cancelled by a back-flow of fluid within the interface. We thus predict a 'phoretic' velocity of magnitude $U \sim-\left(a^{2} / \xi\right) k T \nabla n$, where $a$ is the size of the particle, $1 / \xi$ is the particle's mobility in the interface and $\nabla n$ is the concentration gradient of surfactant in the interface. Note that this velocity is not necessarily small, i.e. not proportional to some small interactive potential length $b$, but rather to the size of the particle $a$.

This work was supported in part by grant 0754967 from the National Science Foundation. 


\section{Appendix. Hydrodynamic functions}

The hydrodynamic mobility functions $\hat{L}(s)$ and $\hat{M}(s)$ are given by

$$
\begin{aligned}
& \hat{L}(s)=\frac{A_{22}-\lambda \nu A_{11}}{1-\lambda \nu}-\frac{2 \lambda(1-v) A_{12}}{(1+\lambda)(1-\lambda \nu)}, \\
& \hat{M}(s)=\frac{B_{22}-\lambda \nu B_{11}}{1-\lambda \nu}-\frac{2 \lambda(1-v) B_{12}}{(1+\lambda)(1-\lambda \nu)},
\end{aligned}
$$

where the $A_{i j}$ and $B_{i j}$ are the standard mobility functions for motion along and transverse to the line of centres of two spheres (see Batchelor 1982). Similarly, the hydrodynamic mobility functions for the relative diffusivity are given by

$$
\begin{aligned}
& G(s)=\frac{A_{22}+\lambda A_{11}}{1+\lambda}-\frac{4 \lambda A_{12}}{(1+\lambda)^{2}}, \\
& H(s)=\frac{B_{22}+\lambda B_{11}}{1+\lambda}-\frac{4 \lambda B_{12}}{(1+\lambda)^{2}} .
\end{aligned}
$$

The hydrodynamic mobility functions $L(s)$ and $M(s)$ are defined by

$$
\hat{\boldsymbol{M}}_{i i}-\hat{\boldsymbol{M}}_{i j}=L(s) \hat{\boldsymbol{s}} \hat{\boldsymbol{s}}+M(s)(\boldsymbol{I}-\hat{\boldsymbol{s}} \hat{\boldsymbol{s}}),
$$

corresponding to motion along, $L$, and transverse, $M$, to the line of centres of the two particles. In terms of the standard mobility functions they are given by

$$
\begin{gathered}
L(s)=A_{11}-\frac{2}{1+\lambda} A_{12}, \\
M(s)=B_{11}-\frac{2}{1+\lambda} B_{12},
\end{gathered}
$$

and

$$
W(s)=\frac{2(L-M)}{s}+\frac{\mathrm{d} L}{\mathrm{~d} s} .
$$

\section{REFERENCES}

Abecassis, B., Cottin-Bizonne, C., Ybert, C., Ajdari, A. \& Bocquet, L. 2008 Boosting migration of large particles by solute contrasts. Nature Mater. 7, 785-789.

Anderson, J. L. 1989 Colloidal transport by interfacial forces. Annu. Rev. Fluid Mech. 21, 61-99.

Asakura, S. \& Oosawa, F. 1954 On the interaction between two bodies immersed in a solution of macromolecules. J. Chem. Phys. 22, 1255-1256.

BAtCheloR, G. K. 1970 Stress system in a suspension of force-free particles. J. Fluid Mech. 41, $545-570$.

Batchelor, G. K. 1972 Sedimentation in a dilute suspension of spheres. J. Fluid Mech. 752, 245-268.

BATCHELOR, G. K. 1976 Brownian diffusion of particles with hydrodynamic interaction. J. Fluid Mech. 74, 1-29.

BAtCheloR, G. K. 1982 Sedimentation in a dilute polydisperse system of interacting spheres. Part 1. General theory. J. Fluid Mech. 119, 379-408.

Batchelor, G. K. 1983 Diffusion in a dilute polydisperse system of interacting spheres. J. Fluid Mech. 131, 155-175.

Berne, B. J. \& Pecora, R. 1976 Dynamic Light Scattering. Wiley.

Bonnecaze, R. T. \& Brady, J. F. 1992 Dynamic simulation of an electrorheological fluid. J. Chem. Phys. 96, 2183-2202.

Brady, J. F. 1993 Brownian motion, hydrodynamics and the osmotic pressure. J. Chem Phys. 98, $3335-3341$.

Brady, J. F. \& Bossis, G. 1988 Stokesian dynamics. Annu. Rev. Fluid Mech. 20, 111-157. 
Brady, J. F., Phillips, R. J., Lester, J. C. \& Bossis, G. 1988 Dynamic simulation of hydrodynamically interacting suspensions. J. Fluid. Mech. 195, 257-280.

Córdova-Figueroa, U. M. 2008 Directed motion of colloidal particles via chemical reactions: Osmotic propulsion. PhD thesis, California Institute of Technology, Pasadena.

Córdova-Figueroa, U. M. \& Brady, J. F. 2008 Osmotic propulsion: the osmotic motor. Phys. Rev. Lett. 100, 158-303.

Ebbens, S. J. \& Howse, J. R. 2010 In pursuit of propulsion at the nanoscale. Soft Matt. 6, 726-738.

Foss, D. R. \& Brady, J. F. 2000 Structure, diffusion and rheology of Brownian suspensions by Stokesian dynamics simulation. J. Fluid Mech. 407, 167-200.

Golestanian, R., Liverpool, T. B. \& Ajdari, A. 2005 Propulsion of a molecular machine by asymmetric distribution of reaction products. Phys. Rev. Lett. 94, 220801.

Golestanian, R., Liverpool, T. B. \& Ajdari, A. 2007 Designing phoretic micro- and nanoswimmers. New J. Phys. 9, 126.

Hong, Y., Blackman, N. M. K., Kopp, N. D., Sen, A. \& Velegol, D. 2007 Chemotaxis of nonbiological colloidal rods. Phys. Rev. Lett. 99, 178103.

Howse, J. R., Jones, R. A. L., Ryan, A. J., Gough, T., Vafabakhsh, R. \& Golestanian, R. 2007 Self-motile colloidal particles: from directed propulsion to random walk. Phys. Rev. Lett. 99, 048102.

Ibele, M., Mallouk, T. E. \& Sen, A. 2009 Schooling behavior of light-powered autonomous micromotors in water. Angew. Chem. Intl Ed. Engl. 48, 3308-3312.

Jülicher, F. \& Prost, J. 2009a Comment on 'osmotic propulsion: the osmotic motor'. Phys. Rev. Lett. 103, 079801.

Jülicher, F. \& Prost, J. 2009 b Generic theory of colloidal transport. Eur. Phys. J. E 29, $27-36$.

Kagan, D., Calvo-Marzal, P., Balasubramanian, S., Sattayasamitsathit, S., Manesh, K. M., Flechsig, G.-U. \& WANG, J. 2009 Chemical sensing based on catalytic nanomotors: motionbased detection of trace silver. J. Am. Chem. Soc. 131, 12802-12083.

KhaIR, A. S. \& Brady, J. F. 2006 Single particle motion in colloidal dispersions: a simple model for active and nonlinear microrheology. J. Fluid Mech. 557, 73-117.

LADD, A. J. C. 1990 Hydrodynamic transport coefficients of random dispersions of hard spheres. J. Chem. Phys. 93, 3484-3494.

Nitsche, J. M. \& Batchelor, G. K. 1997 Break-up of a falling drop containing dispersed particles. J. Fluid Mech. 340, 161-175.

Paxton, W. F., Kistler, K. C., Olmeda, C. C., Sen, A., St. Angelo, S. K., Cao, Y., Mallouk, T. E., LAmmert, P. E. \& Crespi, V. H. 2004 Catalytic nanomotors: autonomous movement of striped nanorods. J. Am. Chem. Soc. 126, 13424-13431.

Paxton, W. F., Sundararajan, S., Mallouk, T. E. \& Sen, A. 2006 Chemical locomotion. Angew. Chem. Intl Ed. Engl. 45, 5420-5429.

Russel, W. B., Saville, D. A. \& Schowalter, W. R. 1989 Colloidal Dispersions. Cambridge University Press.

Squires, T. M. \& Brady, J. F. 2005 A simple paradigm for active and nonlinear microrheology. Phys. Fluids 17, 073101.

Wang, Y., Fei, S.-T., Byun, Y.-M., Lammert, P. E, Crespi, V. H, Sen, A. \& Mallouk, T. E. 2009 Dynamic interactions between fast microscale rotors. J. Am. Chem. Soc. 131, 9926-9927.

VAN DER WerfF, J. C. \& DE KRUIF, C. G. 1989 Hard-sphere colloidal dispersions: the scaling of rheological properties with particle size, volume fraction and shear rate. J. Rheol. 33, 421-454. 